\title{
Microscopic picture of constraint release effects in entangled star polymer melts
}

Article

Accepted Version

Cao, J. and Wang, Z. (2016) Microscopic picture of constraint release effects in entangled star polymer melts.

Macromolecules, 49 (15). pp. 5677-5691. ISSN 1520-5835 doi: https://doi.org/10.1021/acs.macromol.6b00554 Available at https://centaur.reading.ac.uk/66249/

It is advisable to refer to the publisher's version if you intend to cite from the work. See Guidance on citing.

To link to this article DOI: http://dx.doi.org/10.1021/acs.macromol.6b00554

Publisher: American Chemical Society

All outputs in CentAUR are protected by Intellectual Property Rights law, including copyright law. Copyright and IPR is retained by the creators or other copyright holders. Terms and conditions for use of this material are defined in the End User Agreement.

\section{www.reading.ac.uk/centaur}

\section{CentAUR}

Central Archive at the University of Reading

Reading's research outputs online 


\title{
Microscopic Picture of Constraint Release Effects in Entangled Star Polymer Melts
}

\author{
Jing Cao* and Zuowei Wang* \\ Department of Mathematics and Statistics, University of Reading, Whiteknights, PO Box \\ 220, Reading RG6 6AX, UK \\ E-mail: jingcaomath@gmail.com; zuowei.wang@reading.ac.uk
}

\begin{abstract}
The constraint release (CR) effect in entangled branched polymers is generally described by the widely accepted Dynamic Tube Dilation (DTD) theory based on the tube model, which predicts the stress relaxation function reasonably well, but not the dielectric or arm end-to-end vector relaxation. The microscopic picture of entanglement dynamics even in the simple case of star polymers is still not fully resolved. In this work, we first perform molecular dynamics simulations of symmetric star polymer melts using the Kremer-Grest bead-spring model. The entanglement events are analysed microscopically using the persistent close-contacts between mean paths of neighboring polymer strands. The resulted survival probability function of these entanglements or close-contacts show reasonably good agreement with the stress relaxation function, which provides qualitative evidence for the binary picture of entanglements. Based on this understanding we further investigate the star arm retraction and CR effects using the coarse-grained single-chain slip-spring model originally developed by Likhtman and also a simplified single-chain stochastic model. Our simulations revealed that, for
\end{abstract}

\footnotetext{
* To whom correspondence should be addressed
} 
entanglements sitting on a target star arm, only those destroyed by the arm free end dominate the arm end-to-end vector relaxation, while the constraint release events produce an accelerated drift of the mean positions of these specific entanglements towards the arm-end, which is an essential mechanism for understanding the relaxation of star polymers in concentrated solutions or melts. Our findings call for an examination of the microscopic foundation of conventional DTD picture and inspire the development of quantitative theories with consideration of more microscopic details.

\section{Introduction}

The dynamics of entangled polymers has been predominantly described by the tube model for several decades. ${ }^{1-6}$ In this model the topological constraints on a polymer chain are represented by a mean-field potential that confines the chain to a tube-like region. The stress relaxation in a system of entangled linear chains proceeds via three main mechanisms, namely reptation, contour length fluctuation $(\mathrm{CLF})$ and constraint release (CR). Both linear and nonlinear viscoelastic behavior of monodisperse linear polymers with high molecular weights have been successfully described by tube-based theories. ${ }^{3,5-9}$

For branched polymers, such as star, H-, comb and Cayley-tree polymers, reptation dynamics is strongly suppressed by the effectively localized branch points. These polymers thus relax in a hierarchical way, starting from the retraction of the outermost branch arms and proceeding to inner layers till the core of the molecules. The retraction of isolated star arms in a fixed network was first modeled by Pearson and Helfand as the one-dimensional (1D) diffusion of a single particle subject to an effective potential whose magnitude grows exponentially, following the relation of $U(s)=3 Z s^{2} / 2$ where $Z$ is the number of entanglements and $s$ is the relaxed fraction of the arm. ${ }^{10}$ In concentrated solutions or melts of star polymers, constraint release softens the arm retraction potential. Ball and McLeish ${ }^{11}$ treated the CR effects in a self-consistent manner by assuming the relaxed arm segments as a solvent for the unrelaxed materials, which effectively reduces the number of entanglements in the system by 
a factor of $1-s$ and so results in a gradual dilation of the confining tube. The hypothesized relaxation mechanism is generally termed as "Dynamic Tube Dilation"(DTD). ${ }^{11,12}$ Based on this picture, Milner and McLeish ${ }^{13,14}$ developed a theory for predicting the mean first passage (FP) time of arm retraction by solving the Kramers' problem of one bead linked to the origin through a harmonic spring. ${ }^{15}$ After properly including the contributions from early time fluctuations of the arm free end, this theory predicts the loss modulus $G^{\prime \prime}(\omega)$ of symmetric star polymer melts in good agreement with experiments for the whole range of frequencies. Computational models based on a similar tube-theory framework have also been developed for predicting the linear rheology of general mixtures of branched polymers. ${ }^{16-20}$ Recently we have shown by simulations and theoretical calculations that if the star arm is represented by a Rouse chain with more beads, the mean FP time of the chain extension or retraction gets faster than that predicted by the single-bead models. ${ }^{21}$ More accurate description of arm retraction dynamics should be achieved by solving a multi-dimensional Kramer's problem even for the cases without CR.

Concerning the constraint release effects, the DTD model has been shown to describe the viscoelastic behaviors of monodisperse linear and symmetric star polymer melts reasonably well, ${ }^{13,14}$ but encountered difficulty in providing a good description of the dielectric relaxation and terminal stress relaxation of star polymers. ${ }^{3,22-24}$ One reason lies in that this model predicts a tube dilation process faster than the transverse Rouse motion of the arm when the free end retracts close to the branch point. To explain the deviations from the dynamic dilution predictions, Shanbhag et al. ${ }^{25}$ proposed a multi-chain "slip-link" model by assuming entanglements as topological constraints arising from pairs of neighboring chains. Their simulation results suggest that the relaxation in the terminal regime is controlled by the rare events that new entanglements created in between the branch points and deepest entanglements push the original deep entanglements toward the arm ends and so allow them to be deleted by relatively shallow arm retractions. Following this qualitative elucidation of the deviation from the DTD theory, several questions could be raised: 1) can one verify the 
mechanism revealed by the simplified slip-link model in a more realistic model of entangled polymers? 2) if this mechanism exists, does it only contribute to the dynamics of the deepest entanglements? We will tackle these questions in the current work using a multi-scale simulation approach.

The aim of this paper is to provide a microscopic picture of how CR affects the entanglement dynamics in star polymer melts. First we carry out molecular dynamics (MD) simulations of melts of symmetric star polymers represented by the flexible Kremer-Grest model ${ }^{26}$ and perform contact map analysis in which entanglements are defined by persistent binary contacts between neighboring arms. ${ }^{27}$ We find that the stress relaxation function $G(t)$ can be reasonably well reproduced by the survival probability function of these entan-

glements or persistent close-contacts. Based on this binary picture of entanglements, the single-chain slip-spring model developed by Likhtman ${ }^{28}$ is confidently used as a computationally efficient way to study the dynamics of entanglements (slip-links) to much larger time and length scales. The slip-spring model also allows the control of CR effects. We find that the dielectric or end-to-end vector relaxation function of star arms is determined by the slip-links destroyed from the arm-ends and CR facilitates a drift of the mean positions of these specific slip-links towards the corresponding arm-ends. In order to find out the essential physical origins of the observed drifting behavior of entanglements we propose a more coarse-grained single-chain stochastic model for separating different elements that affect the motion of entanglements along the arms. In the end, discussion and conclusion sections follow.

\section{Determining entanglements in MD simulations}

To examine the existing theories as well as resolve some long-standing puzzles, molecular dynamics simulation has been introduced as a suitable methodology to study the dynamics of entangled polymers in melts, since we can visualize the conformations and trajectories of 
individual polymers and their neighbors. ${ }^{26,27,29-44}$ Because of the slow relaxation of branched polymers and limited computational power, the number of entanglement strands per star arm studied in MD simulations are typically smaller than those used in experiments. But MD simulations can provide us the details about what exactly happens at microscopic length and time scales, which are generally inaccessible to experiments. They can thus be treated as computational experiments, offering more information for the development of more coarsegrained models and theories for illustrating entanglement dynamics.

\subsection{Kremer-Grest Model}

In this work, monodisperse 3-arm symmetric star polymer melts are simulated using the flexible Kremer-Grest (KG) bead-spring model. ${ }^{26}$ The excluded volume interaction between monomers is represented by a purely repulsive Lennard-Jones(LJ) potential with the potential parameter $\epsilon=k_{B} T$ and a cutoff distance $r_{c}=2^{1 / 6} \sigma$ where $\sigma$ is the diameter of the monomers. The time unit is given by $\tau_{L J}=\left(m \sigma^{2} / \epsilon\right)^{1 / 2}$ with $m$ the monomer mass. Finitely extensible nonlinear elastic(FENE) potential is introduced between bonded neighbours with the spring constant $k=30 \epsilon / \sigma^{2}$ and the maximum of bond length $R_{\max }=1.5 \sigma$. All simulations are performed in the canonical ensemble with temperature $T=1$ and monomer number density $\rho=0.85 \sigma^{-3}$. In the simulation box there are $N_{c}=70$ star molecules with the number of beads per arm $N=255$. Every single run of the simulations has reached approximately $100 \tau_{d}$ where $\tau_{d} \approx 2 \times 10^{6} \tau_{L J}$ is the terminal stress relaxation time of the stars. Therefore the samples are well equilibrated and the trajectories are long enough for carrying out the binary close-contact analysis. ${ }^{27}$ The entanglement molecular weight $N_{e}$ in such flexible KG chain systems was found to be $N_{e}^{P P} \approx 65$ from primitive path analysis ${ }^{45}$ and $N_{e}^{M S D} \approx 50$ from the monomer mean square displacements (MSD), ${ }^{31}$ respectively. The Rouse relaxation time of an entanglement strand $\tau_{e}$ was estimated to be around $3000 \tau_{L J}$ by using the crossover between the MSD data in the Rouse and entangled regimes. ${ }^{31}$ All the time correlation functions, including the stress and dielectric relaxation functions, are calculated on the fly using 
the multiple-tau correlator method to ensure good statistics. ${ }^{46}$

\subsection{Constructing primitive paths of star polymers}

To examine the tube picture in MD simulations, the first challenge we face is how to define the tubes or primitive paths. One approach is the primitive-path analysis method developed by Everaers et al., ${ }^{45,47}$ in which the primitive paths of entangled chains are procured by fixing the chain ends in space, switching off the intra-chain excluded-volume interactions and then minimizing the energy. Since the interchain interactions are still preserved, the shrunk chains cannot cross each other and so have to take piecewise linear conformations between binary entanglement points. Similar chain-shrinking methods have also been developed by Kröger ${ }^{48}$ and Theodorou et al. ${ }^{49,50}$ who used geometric algorithms to straighten chain paths and hence abridge chain contour lengths without allowing chains to cross. Zhou and Larson have examined both the total energy minimization and the length minimization methods in MD simulations of the KG chains. ${ }^{51}$ Clearly, the chain-shrinking process destroys the local structure of the melts such that the details of the tube cannot be observed. Later two different approaches without destroying the local structures were proposed, which are the isoconfigurational ensemble $(\mathrm{ICE})^{36}$ and the mean path $(\mathrm{MP})^{52}$ methods. In the ICE method, multiple short MD simulations are performed starting with the same initial chain conformations but different monomer initial velocities. The primitive paths of the chains are obtained by averaging over different trajectories and over a short averaging time. In the MP method, the primitive paths consist of the mean position of each bead over some averaging time $\tau_{a v}$ during a single long MD run. Both methods can produce smooth primitive paths, because fast fluctuations and small length scale wiggles are eliminated by the averaging procedure. Considering the computational convenience and efficiency, we employ the MP method for entanglement analysis of the star polymers studied in this work. 


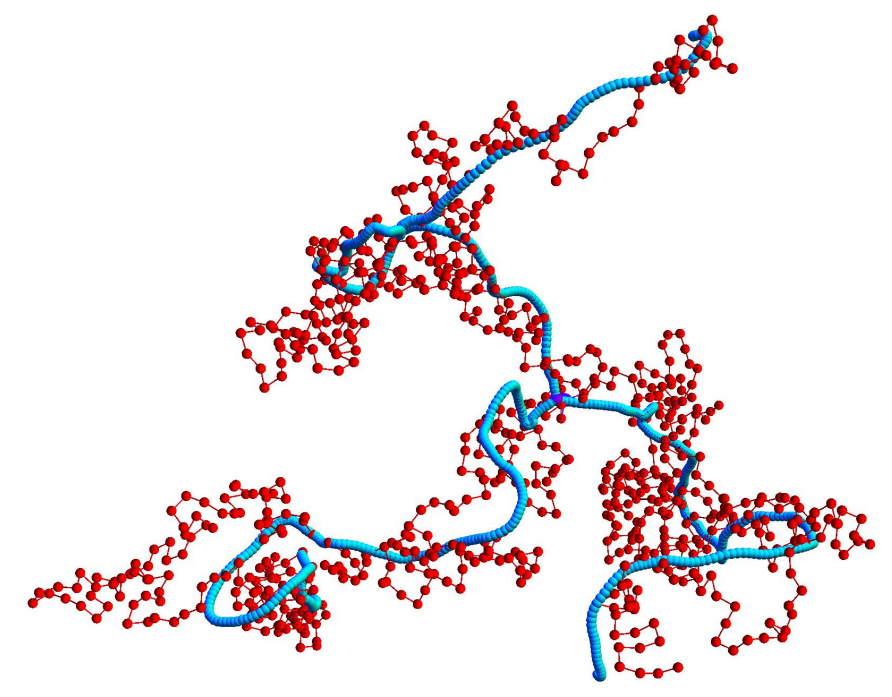

Figure 1: Instantaneous conformation (red) and the mean path calculated with an averaging time $\tau_{a v}=12000 \tau_{L J}($ light blue) of a symmetric 3 -arm star polymer studied in the MD system.

The mean position of the $i$-th monomer in a given polymer chain is defined as

$$
\hat{\boldsymbol{r}}_{i}(t)=\frac{1}{\tau_{a v}} \int_{t-\tau_{a v}}^{t} \boldsymbol{R}_{i}\left(t^{\prime}\right) d t^{\prime}
$$

where $\boldsymbol{R}_{i}\left(t^{\prime}\right)$ is the instantaneous position of this monomer at time $t^{\prime}$. The mean path of the corresponding chain thus consists of the mean positions of all its constituent monomers. The exact shape of the mean path depends on the choice of the averaging time $\tau_{a v}$. With increasing $\tau_{a v}$, the path becomes smoother, as fluctuation modes with characteristic times shorter than $\tau_{a v}$ are averaged out. On the other hand, it is also desirable to keep the mean path as local as possible both in space and time. Therefore, the value of $\tau_{a v}$ is typically taken to be of the order of the entanglement time $\tau_{e}$. This is the timescale needed for a chain segment to explore the confining tube transversely. More detailed discussions of mean path analysis can be found in Refs. ${ }^{52}$ and. ${ }^{27}$ Fig. 1 shows the MP of a symmetric 3arm star (light blue) calculated with $\tau_{a v}=12000 \tau_{L J} \approx 3 \tau_{e}$, together with its instantaneous configuration (red). 


\subsection{Persistent close contacts between mean paths}

The entanglements can be defined by persistent close-contacts between mean paths of pairs of neighboring chains as originally described in Ref. ${ }^{27}$ Here, we briefly summarize the approach. A contact map is created for each pair of chains $\left(j_{1}, j_{2}\right)$ in the system to quantify entanglements over time. If the mean position of monomer $i_{1}$ of chain $j_{1}$ is within a certain distance $d_{c u t}$ from the mean position of monomer $i_{2}$ of chain $j_{2}$ at time $t$, a point $\left(i_{1}, i_{2}, t\right)$ will be appended to the three-dimensional (3D) contact map. Furthermore, if two contacts are less than $i_{c u t}$ monomers away from each other along a given chain and are in the same or the adjacent time frames, they are grouped into one cluster. We choose $i_{c u t}=30 \approx N_{e} / 2$ which by observation is the maximum number of monomers a chain can slide between adjacent time frames. Each independent cluster is called an entanglement. Note that each cluster contains more than one contact. An algorithm was developed to process each cluster of contacts in order to return one monomer pair $i_{1}(t)$ and $i_{2}(t)$ for each frame, indicating which monomers are involved in an entanglement at a particular time. In the end, a smooth trajectory of $\left\{i_{1}(t), i_{2}(t), t\right\}$ can be obtained for each entanglement in the system, see Fig.2 below or Fig.4 in Ref. ${ }^{27}$ The mean path averaging time $\tau_{a v}$ and the cutoff distance $d_{c u t}$ are the two main parameters for the contact map algorithm, which are set to be $12000 \tau_{L J}$ and $3 \sigma$ in this work, respectively.

In the original version of the contact map algorithm, ${ }^{27}$ the close-contact is required to exist at every moment for determining the lifetime of the corresponding entanglement. As a result, the contact trajectories are always continuous in time. However, as we know, for some less strongly interacting primitive paths, thermal fluctuations of tension could be sufficient to engage and disengage their close-contacts, depending on whether the distances between them are within the cut-off threshold or not. ${ }^{39}$ Indeed, any persistent close-contact defined through a distance threshold suffers from this problem. For the purpose of illustration, Fig.2 shows one example of two smooth contact trajectories between a pair of star arms as determined using the original algorithm. As marked by the dashed circle, there is no contact detected in 


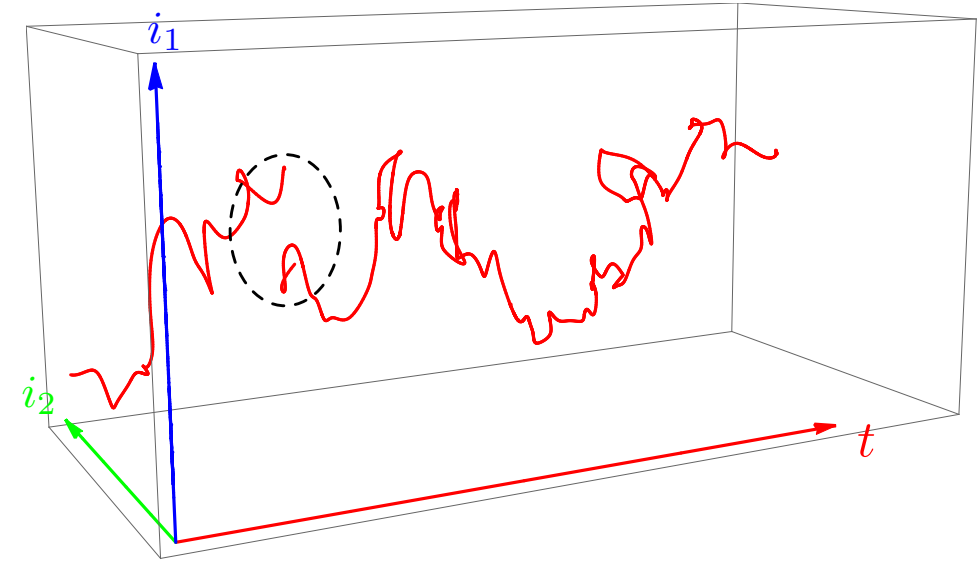

Figure 2: Trajectories of persistent close-contacts between a pair of star arms in the MD system as determined using the original contact map algorithm. The blue and green axes indicate the monomer indices on the two different arms involved in the contacts.

one particular time frame, because the distance between the two mean paths exceeds $d_{c u t}$. This is probably due to the involvement of a third arm which temporarily pushes the two arms away from each other. But in the next time frame the two arms get into contact again in the proximity of the previous contact point. The two separated contact trajectories should actually be linked together to represent one entanglement. In order to diminish the defect in the distance threshold criterion, we update the contact map algorithm by deliberating that if a close-contact disappears in a time frame where neither of the involved segments is at the corresponding chain end and then reappears in the contact map after a short time $\tau_{\text {gap }}$ near the location where it was destructed, its trajectory will be treated as continuous over that period of time and so represents one single entanglement.

\subsection{Properties of persistent close contacts (entanglements)}

At time scales much smaller than the Rouse time $\left(t<<\tau_{R}\right)$, the arm free end experiences fast fluctuations under the action of many Rouse modes. ${ }^{5,13}$ Theoretical models based on the solutions of Kramer's problems are not applicable for describing such shallow arm retraction, because the effective energy barrier is lower than $k_{B} T$. In this work we will mainly focus on 
the deep or late-time arm retractions which determine the terminal relaxations. Therefore only the persistent close-contacts that live longer than $10 \tau_{a v} \approx 30 \tau_{e} \approx \tau_{R}$ are collected for entanglement analysis. The long MD simulations runs (up to $100 \tau_{d}$ ) ensure us to have sufficient number of close-contact samples even for entanglement lifetimes beyond the stress relaxation terminal time $\tau_{d}$.

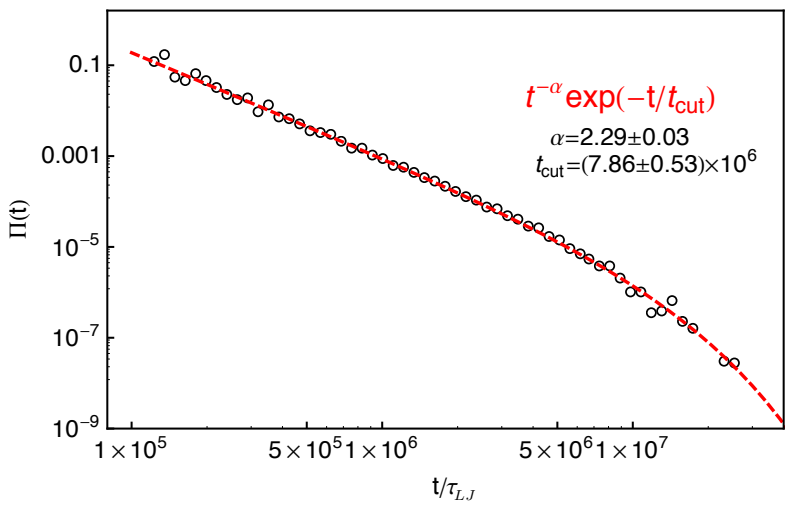

Figure 3: Probability density $\Pi(t)$ that a randomly selected entanglement has a lifetime $t$ (symbols) and the best fitting function with the expression $t^{-\alpha} \exp \left(-t / t_{\text {cut }}\right)$ (line).

The probability density $\Pi(t)$ of a randomly chosen entanglement having a lifetime $t$ is shown in Fig.3, which can be reasonably well described by a function of a power-law decay with an exponential cutoff,

$$
\Pi(t) \sim t^{-\alpha} \exp \left(-\frac{t}{t_{c u t}}\right)
$$

where $\alpha \approx 2.29$ and $t_{c u t} \approx 7.86 \times 10^{6} \tau_{L J}$. The data in Fig.3 have been properly normalized such that the integral of $\Pi(t)$ over the entire time range is equal to 1 . We note that $\Pi(t)$ basically represents the spectrum of CR events, which could be potentially used for developing quantitative theories or single-chain simulation models for entangled star polymers. This function decays fast with time, indicating that the majority of CR events take place at relatively short time scales. On the other hand, for predicting the dynamic and rheological properties, such as the stress, birefringence or dielectric relaxations, which are typically time 
correlation functions, the more relevant quantity is the survival probability $P_{\text {ent }}(t)$ that an entanglement picked randomly at a given time will still exist after certain elapsed time $t .2,53$
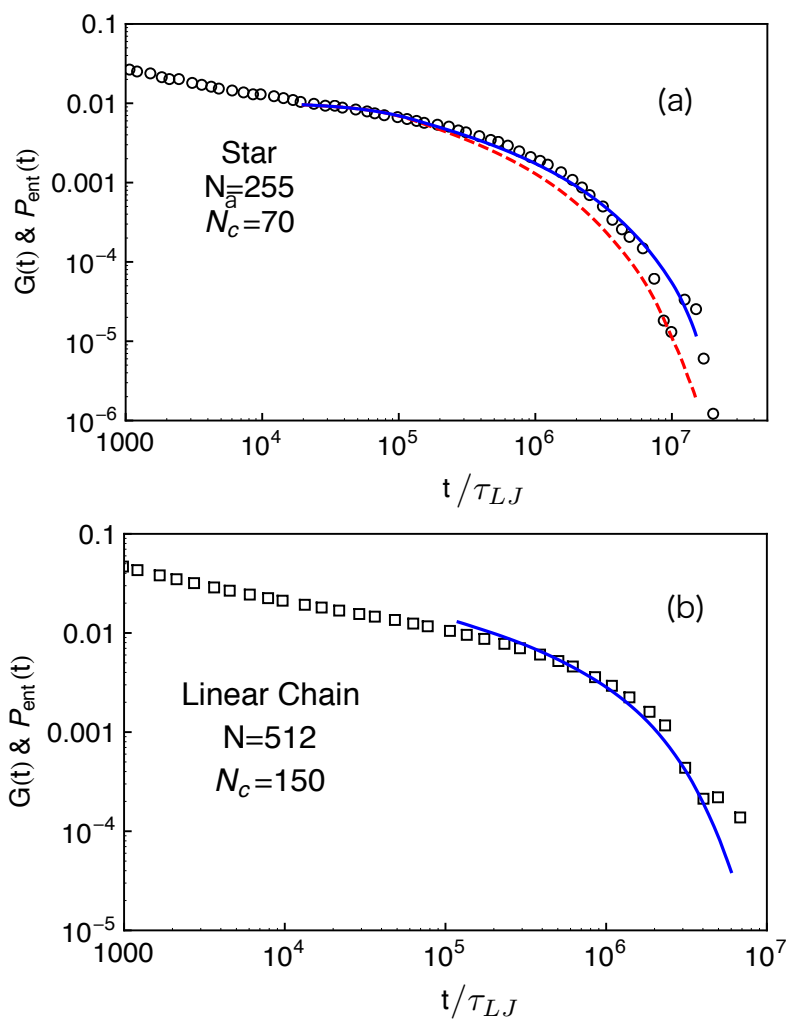

Figure 4: (a) Comparison between stress relaxation function $G(t)$ (symbols) and entanglement survival probabilities $P_{\text {ent }}(t)$ for symmetric stars of arm-length $N=255$ (dashed line: original close-contact algorithm in ref., ${ }^{27}$ solid line: updated algorithm introduced in this work). (b) Same comparison as in (a) but for linear chain melt system with chain length $N=512$.

There is a simple relationship between $P_{\text {ent }}(t)$ and $\Pi(t)$,

$$
P_{\text {ent }}(t)=\frac{\int_{t}^{+\infty}(\tau-t) \Pi(\tau) d \tau}{\int_{0}^{+\infty} \tau \Pi(\tau) d \tau}
$$

The $P_{\text {ent }}(t)$ results obtained using the updated contact map algorithm are shown in Fig.4(a) by the blue solid line, while those generated with the original algorithm in ref. ${ }^{27}$ are shown by the red dashed line. The former set of data show much better agreement with the stress relaxation function $G(t)$ of the system. It should be noted that in the calculation of $P_{\text {ent }}(t)$ 
all entanglements have been taken into account, which consequently includes contributions from both arm-retraction and constraint release. We also find the shapes of $P_{\text {ent }}(t)$ depend imperceptibly on the frame gap $\tau_{g a p}$ if $\tau_{g a p} \geq 2 \tau_{a v} \approx 6 \tau_{e}$. Therefore, $\tau_{g a p}$ used in the updated contact map method is set to $2 \tau_{a v}$. For completeness, the close-contact analysis using the updated version is also carried out in a linear chain melt system with $N=512$ and $P_{\text {ent }}(t)$ is again in a reasonable agreement with $G(t)$ as shown in Fig.4(b). These results indicate that the binary contact picture can be applied to describe the entanglement dynamics in both linear and star polymer melts.

Furthermore we have monitored the relative ordering of the entanglements along the same arm. The crossover rate between neighboring entanglements is found to be less than $3 \%$ per entanglement per time frame $\left(3 \tau_{e}\right)$. In other words, a given entanglement only swaps with its neighbors once every $100 \tau_{e}$. Considering that this swapping rate has also counted in the reverse crossing events between neighboring entanglements, the long-term or permanent changes of entanglement ordering along the arm are very rare. Therefore in the single-chain slip-spring model used in the following section, effective excluded volume interactions between slip-links are introduced to prevent them from passing over each other or occupy the same monomer. The influence of the non-zero swapping rate on the dynamics of entanglements is discussed in Sec. 5.3.

\section{CR effect studied using single-chain slip-spring model}

Based on the binary picture of entanglements explored in our MD simulations and previous works ${ }^{27,38,45}$ and the fact that $G(t)$ can be reproduced reasonably well by the survival probability of the so-obtained entanglements, the coarse-grained single-chain slip-spring model developed by Likhtman and coworkers for linear polymers $28,31,54,55$ is extended to the simulation of entangled star polymers for accessing time and length scales beyond MD simulations. In this model, the star arms are represented by Rouse chains in 3D space, each consisting 
of $N$ beads with one end bead $(i=N)$ connected to the branch point and the other end $(i=1)$ free to move. The bead friction coefficient is taken to be $\xi=1$ and the average spring length is $b=1$. The topological constraints due to entanglements are modeled by a set of virtual springs of $N_{s}^{s s}$ beads each. One end of each virtual spring is connected to a Rouse monomer by a slip-link, while the other end (anchor point) fixed in space. On average there is one slip-link per chain segment of $N_{e}^{s s}$ monomers. The slip-links move along the arms discretely by hopping from one bead to one of its nearest neighbors with the acceptance rate controlled by a Metropolis Monte-Carlo scheme. Furthermore, each slip-link is paired with another slip-link sitting on an arm of another star. There are thus no self- or intra-arm entanglements considered. To properly incorporate the self-entanglement effects, information on the probability for the polymer arms or chains to be self-entangled would be needed from more detailed simulations such as the Kremer-Grest model via contact map analysis. This will be left for future study. For the arm lengths studied in this work, the contributions from self-entanglements should be relatively small. The exclusion of such effect will not change the results qualitatively.

In our model the creation or destruction of a slip-link always involves at least one arm free end. If a slip-link is deleted from the free end of one arm, its associated partner is also deleted regardless of its location. A new pair of coupled slip-links will be created immediately with one at the end of a randomly chosen arm and the other attached to a monomer on any other arm with equal probability. The total number of slip-links in the system remains constant. The slip-links on the same arm are not allowed to pass over each other or cohabit the same monomer. This effective exclude volume interaction between slip-links is qualitatively consistent with the low swapping rate between neigbhoring entanglements as found in the close-contact analysis in MD simulations. In the current work, the slip-spring model parameters are set to be $N_{e}^{s s}=4$ and $N_{s}^{s s}=0.5$ as have been used in several previous works. ${ }^{31,54,56,57}$ To map the slip-spring simulation data on linear polymer melts obtained using these parameters to the tube model predictions requires the use of $N_{e} \approx 5.7$ beads 
per entanglement. ${ }^{56}$ When mapping the chain middle monomer mean square displacement data generated by this slip-spring model to the MD results obtained using the Kremer-Grest model, a time scaling factor of 3370 was needed. ${ }^{31}$

Each arm is capped at the branch point such that the slip-links cannot slide through to any other arms in the same star. The branch points are fixed in space instead of fluctuating around. Masubuchi et al. have shown in primitive chain network simulations that for asymmetric star polymers the simulations without branch point fluctuations predicted much slower stress relaxation than those with fluctuations. ${ }^{58}$ We have observed a similar but probably less significant effect for symmetric star polymers, as reflected in the comparison between the stress relaxation functions $G(t)$ obtained in the slip-spring and MD simulations in Fig. 5. Different from the MD case where both the intra- and inter-chain contributions are automatically included in the stress calculation, the $G(t)$ results in the single-chain slipspring model simulations are obtained from the single-chain auto-correlation functions to avoid the complication of finding direct physical link between the cross-correlation contributions in the two different models. ${ }^{59,60}$ It has been found in previous simulations that in both models the stress and orientation auto-correlation functions are proportional to the total stress relaxation function at large time scales, especially when close to the terminal time. ${ }^{30,59}$ We can thus use either the single-chain stress or orientation auto-correlation function to represent the $G(t)$ data, with all three functions simply subject to a vertical shift. Apart from the vertical shift, Fig. 5 shows that the slip-spring timescales need to be multiplied by a factor of 1000 for matching the MD time. This time rescaling factor is apparently smaller than that (3370) used in linear polymer cases, ${ }^{31}$ and so reflects the slow dynamics predicted by fixing the branch points. On the other hand, the qualitative agreement between the slip-spring and MD data indicates that at least for the arm lengths studied in the current work, the option of allowing the branch point to fluctuate or not in the slip-spring model will not alter the results qualitatively. Therefore we choose to fix the branch points in space for the convenience of analyzing the slip-link motion along the arms and also being consistent 
with previous slip-link model simulations, ${ }^{25}$ but bearing in mind that the quantitative results might be different by allowing them to fluctuate.

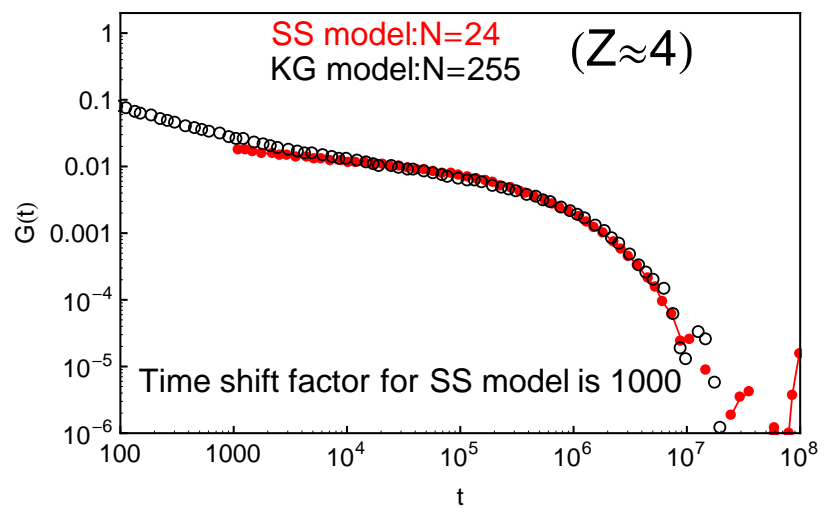

Figure 5: Comparison of stress relaxation functions $G(t)$ obtained in the slip-spring model and MD simulations. The slip-spring data have been shifted both vertically and horizontally, see main text. The number of entanglements per arm is around 4 in both cases.

\subsection{Mean first passage time of tube segment destructions}

In the slip-spring model, constraint relase effect can be easily switched off by decoupling the association between paired slip-links as well as constructing and destructing of slip-links only at arm-ends. Thus, the release of all slip-links on a given arm only relies on arm-retraction, which is analogous to isolated star polymers confined in a permanent network. ${ }^{10,21,61}$ In such a non-CR system, consider a slip-link which sits on monomer $i$ of an arm and so is a fractional distance $s=i / N$ away from the arm-end at a certain time $t$. If this slip-link is deleted from the system at a later time $t^{\prime}$ when it reaches the free end, the first passage time for the arm-end to reach the given slip-link can be simply calculated as $\tau_{n c r}(s)=t^{\prime}-t$.

In the systems with $\mathrm{CR}$, the collection of the FP times $\tau_{c r}(s)$ needs more careful consideration, because each entanglement corresponds to a pair of coupled slip-links sitting on two different arms. An entanglement is deleted from the system when one of the two slip-links is reached by the free end of the arm it sits on. We label the slip-link reached by its corresponding arm-end as $s_{r e t}$ and the other one as $s_{r e f}$. Fig.6(a) shows the schematic conformation of a 


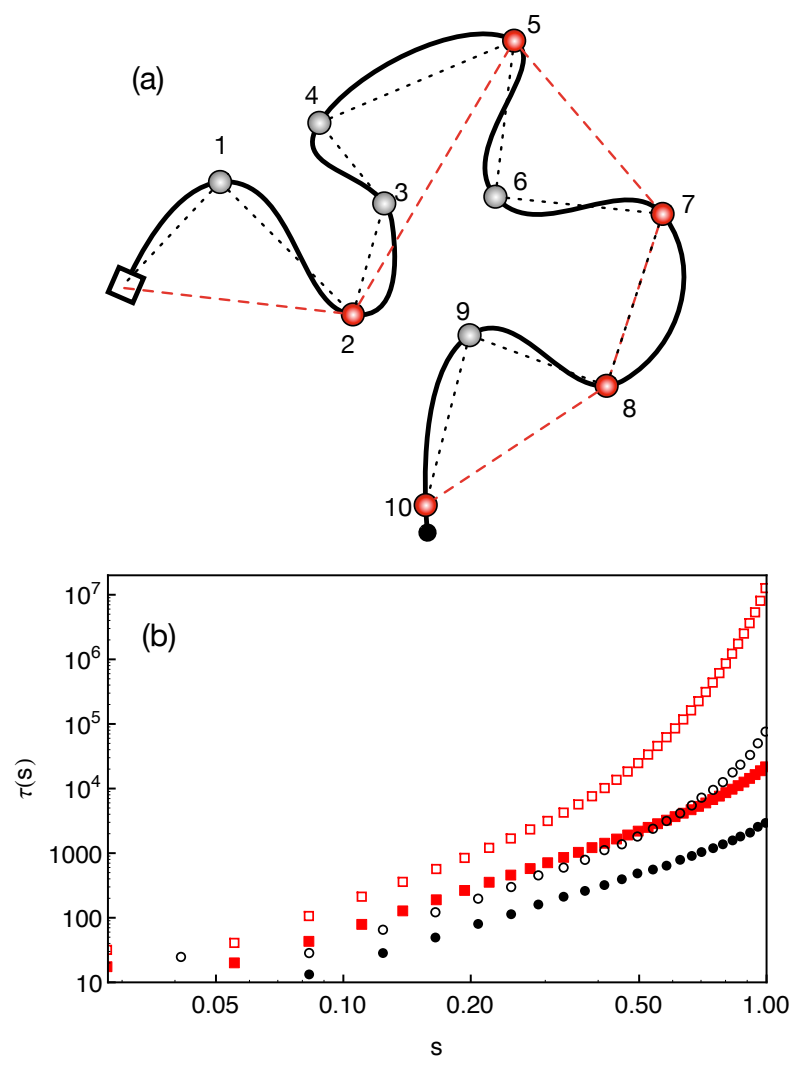

Figure 6: (a) Schematic conformation of a target arm at initial time. Slip-links colored red are the ones that will be destructed by free end of the given arm, while the gray ones will be destructed due to the deletion of their coupled partners elsewhere. (b) Mean first passage times $\tau_{n c r}(s)$ (open, without CR) and $\tau_{c r}(s)$ (filled, with CR) obtained in slip-spring model simulations with arm-length $N_{a}=36$ (red squares) and 24 (black disks), respectively. 
target arm at initial time $t$, in which the open square and black disk indicate the branch point and the arm-end, respectively. The slip-links on this arm are classified into two categories, depending on their fates. The ones destroyed by the end of the given arm $\left(s_{\text {ret }}\right)$ are colored red, while those removed due to the deletion of their coupled partners on some other arms $\left(s_{\text {ref }}\right)$ are colored gray. Considering that the arm is confined in a tube constructed by the slip-springs, the conformation of the tube segment associated with a slip-link marked as $s_{\text {ret }}$ will be completely forgotten after its destruction at $t^{\prime}$. The arm-end can poke out towards any directions from this point. But the tube segment associated with a slip-link marked as $s_{\text {ref }}$ (e. g., slip-link 6 in Fig.6(a)) will only experience a CR event when it is removed. Since the nearest neighboring slip-links of $s_{r e f}$ on the same arm are still present (e. g., slip-links 5 and 7 in relevance to 6 ), the orientation of the original tube segment (i. e., the vector between slip-links 5 and 7) is not relaxed at all. Therefore, for determining the arm end-to-end vector (not stress) relaxation, we can reasonably infer that the tube segment survival times are only associated with the FP times that the arm free end reaches the red or $s_{\text {ret }}$ slip-links. These slip-links are thus termed as "Tube Representative"(TR) slip-links. Note that the TR slip-links on the same arm are released sequentially during the arm retraction process. As will be discussed latter, the CR events contribute to the arm end-to-end vector relaxation by speeding up the release of the TR slip-links from the arm free ends.

Fig.6(b) shows the mean first passage times of TR slip-links in the systems both with and without CR. As we expected, CR speeds up the destruction of the slip-links dramatically. For example, the FP times of the deepest slip-links, or equivalently the terminal relaxation times, in the two systems with arm length $N=36$ or number of entanglements $Z \approx 6$ differ by 3 orders of magnitude. As shown below, our single-chain slip-spring model provides a convenient way for examining the suggestion of Shanbhag et al. on explaining the release of the originally deepest entanglements. ${ }^{25}$ 


\subsection{Dynamics of "Tube Representative" slip-links}

Before presenting any measurements in the slip-spring simulations, we first sketch a qualitative picture about the release of a target TR slip-link which sits next to the branch point of a given arm and will finally be deleted from the arm free end. With CR switched on, new slip-links with lifetimes extracted from a broad distribution (see, e.g., Fig. 3) are incessantly created on the arm at certain rate. These new slip-links have equal probability to be inserted at any locations along the arm. Obviously, the probability to create a new slip-link with lifetime longer than the remaining lifetime of the target TR slip-link on the segment between the branch point and the target one is non-zero during any period of time. After one such insertion, the target TR slip-link feels the new slip-link "permanently" until itself being deleted from the free end. With the simulation going on, more and more such new relatively long-lived slip-links could be inserted in between the branch point and the target slip-link such that the mean position of the target one is gradually pushed away from the branch point. As the target slip-link moves closer to the arm free end, its remaining lifetime is getting shorter and the time threshold for newly inserted slip-links to be considered as "long-lived" with respect to the target slip-link is reduced. The combined effects will lead to a drifting behavior of the target TR slip-link towards the arm-end under an "acceleration field". We note that the term of "long-lived" slip-links used in the current work only refers to the slip-links that live beyond the remaining lifetime of a target slip-link. This definition does not depend on the actual lifetimes and the eventual fates of these slip-links (either deleted by the arm end or by CR events) as long as they are removed from the system after the destruction of the target TR slip-link. According to this definition, any TR slip-link is a "long-lived" one for all other slip-links between itself and the arm-end.

To illustrate the hypothesized picture, Fig.7 shows the trajectory of a target (originally deepest) TR slip-link along a star arm with $N_{a}=36$ as obtained in the slip-spring model simulation. During the lifetime of the target slip-link, all other slip-links sharing the same arm are tracked. For studying the motion of the slip-links along the arm, their locations are 


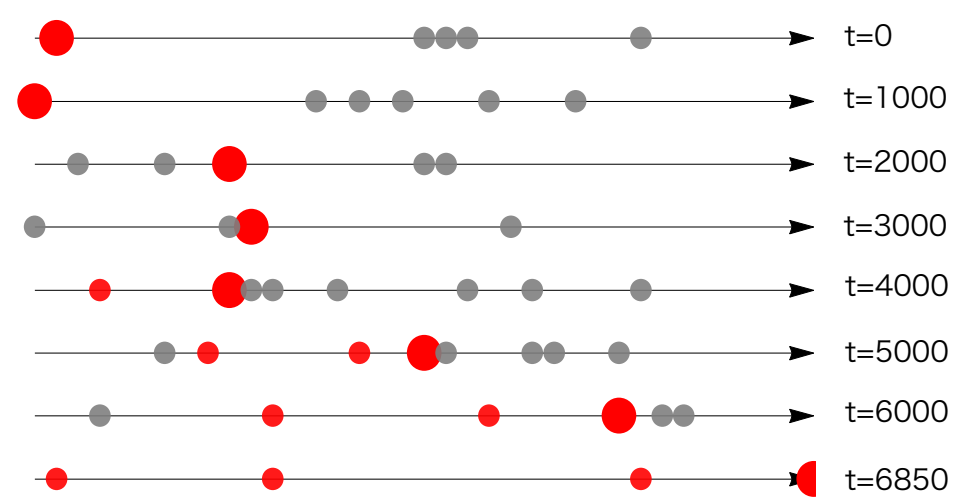

Figure 7: Trajectory of a target (originally deepest) TR slip-link (large red disk) along a star arm with $N_{a}=36$ studied as in the slip-spring model simulation. The "long-lived" and "short-lived" slip-links with regard to the remaining lifetime of the target TR slip-link are represented by the small red and gray disks, respectively. The arrow points from the branch point to the arm-end.

measured by the indices of the monomers that they are attached to, instead of their 3D real space coordinates. Slip-links other than the target one are classified as "long-lived" (red) and "short-lived" (gray) in relation to the remaining lifetime of the target slip-link. Fig.7 shows the propagation of the location (monomer index) of the target TR slip-link along the arm, while the real physical location of the slip-link is always around its anchor point which is fixed in space. Apparently all other slip-links are "short-lived" at the initial time, because the target slip-link is the originally deepest one and will eventually be deleted from the arm-end. As the simulation running, slip-links are continuously created on or deleted from the arm. At later time, some "long-lived" slip-links are created in between the target one and the branch point such that the target slip-link will never be any closer to the branch point as at initial. In the melts of symmetric star polymers with arm length $N_{a}=36$, the ensemble-averaged number of "long-lived" slip-links that are added before the final destruction of the target slip-link is found to be around 3.

Fig.8(a) shows the evolution of the number of slip-links $N_{\text {ent }}$ between the branch point and the target (originally deepest) TR slip-link. Since the lifetime $t^{*}$ of the target slip-link varies from one simulation sample to another, we have normalized the simulation time by 

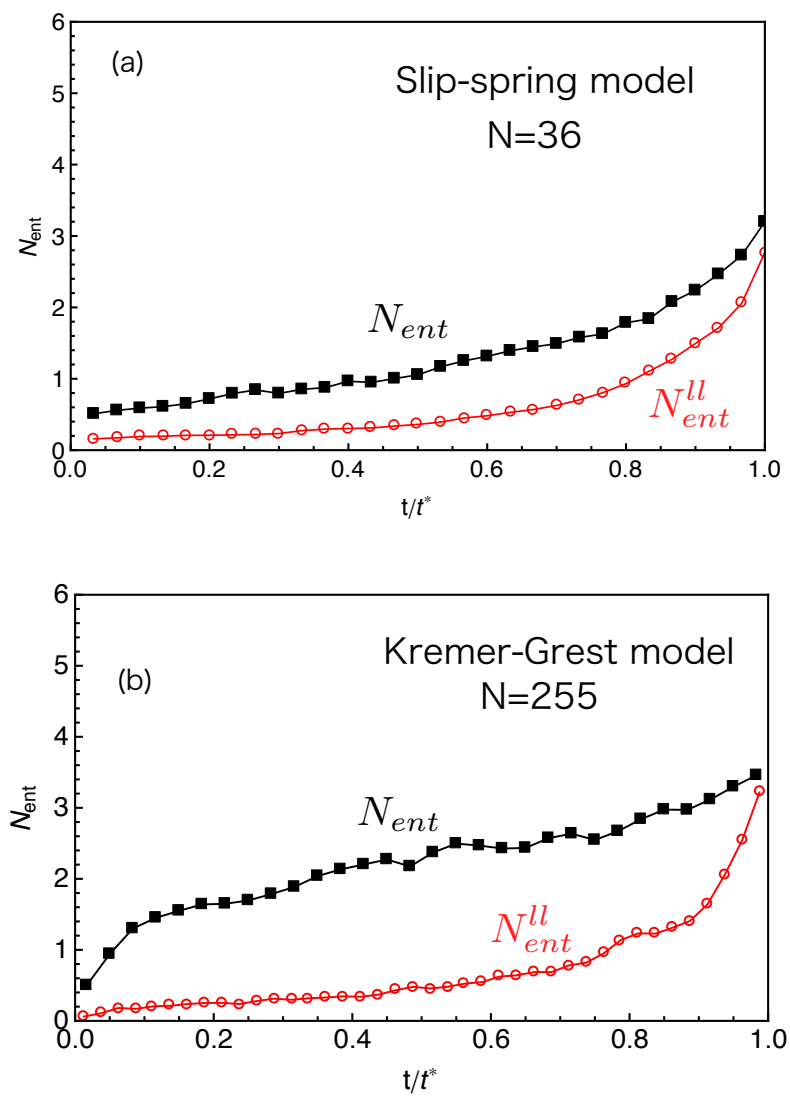

Figure 8: (a) Time-dependent average number of entanglements (slip-links), $N_{\text {ent }}$ (black squares), on the arm segment between the branch point and originally deepest target TR entanglement and average number $N_{e n t}^{l l}$ (red circles) of the "long-lived" ones among them that survive beyond the remaining lifetime of the target one, as obtained in slip-spring model simulations. (b) Same measurements as in (a), but obtained in the contact map analysis of MD simulations using the Kremer-Grest model. The time scale has been normalized by the individual lifetime $t^{*}$ of the target TR entanglement in each case. 
the individual $t^{*}$ value in each case so as to obtain the ensemble average over a dimensionless time period starting from the initial time $t / t^{*}=0$ to the terminal time of the system $t / t^{*}=1$. We can see that $N_{\text {ent }}$ increases monotonically with time, which demonstrates that new sliplinks are continuously created on the segment between the branch point and the target TR slip-link. Among the $N_{e n t}$ slip-links, there are $N_{e n t}^{l l}$ "long-lived" ones with regard to the remaining lifetime of the target slip-link. $N_{\text {ent }}^{l l}$ first shows a slower increase with time than $N_{\text {ent }}$, but eventually reaches the same value as the latter, because upon the destruction of the target TR slip-link, all remaining slip-links on the given arm are "long-lived".

Moreover, we carry out the same measurements in the MD simulations using the persistent close-contact analysis discussed in the previous section. As shown in Fig.8(b), the MD results are in qualitatively good agreement with those obtained in the slip-spring model simulations. It should be noted that the average number of close-contacts (entanglements) on each arm is around 6 for the arm-length of $N=255$. At the moment when the originally deepest entanglement is destructed from the arm free end, there are around $2-3$ entanglements left on the arm. This is consistent with the observation of Shanbhag et al. ${ }^{25}$ that the initially deepest slip-link is pushed about $1 / 3$ of the primitive path towards the arm-ends and so deleted by relatively shallow arm-retractions. To our knowledge, it is the first convincing evidence in the microscopic MD simulations to support the relaxation picture proposed by Shanbhag et al.

We can further investigate the CR effect on the propagation of entanglements along the primitive paths by comparing the temporal variation of the locations of TR slip-links in the systems with and without constraint release. Note that in the systems without CR, all slip-links are considered to be TR ones, since their creation and destruction only happen at the arm free ends. In order to eliminate the direct influences from the reflecting boundary at the branch point and the absorbing boundary at the arm-end, we only choose to study the TR slip-links that sit exactly on the middle monomers $(i=N / 2)$ at the initial time $t=0$. The variation of the locations of these slip-links along the arms, as measured by the monomer 

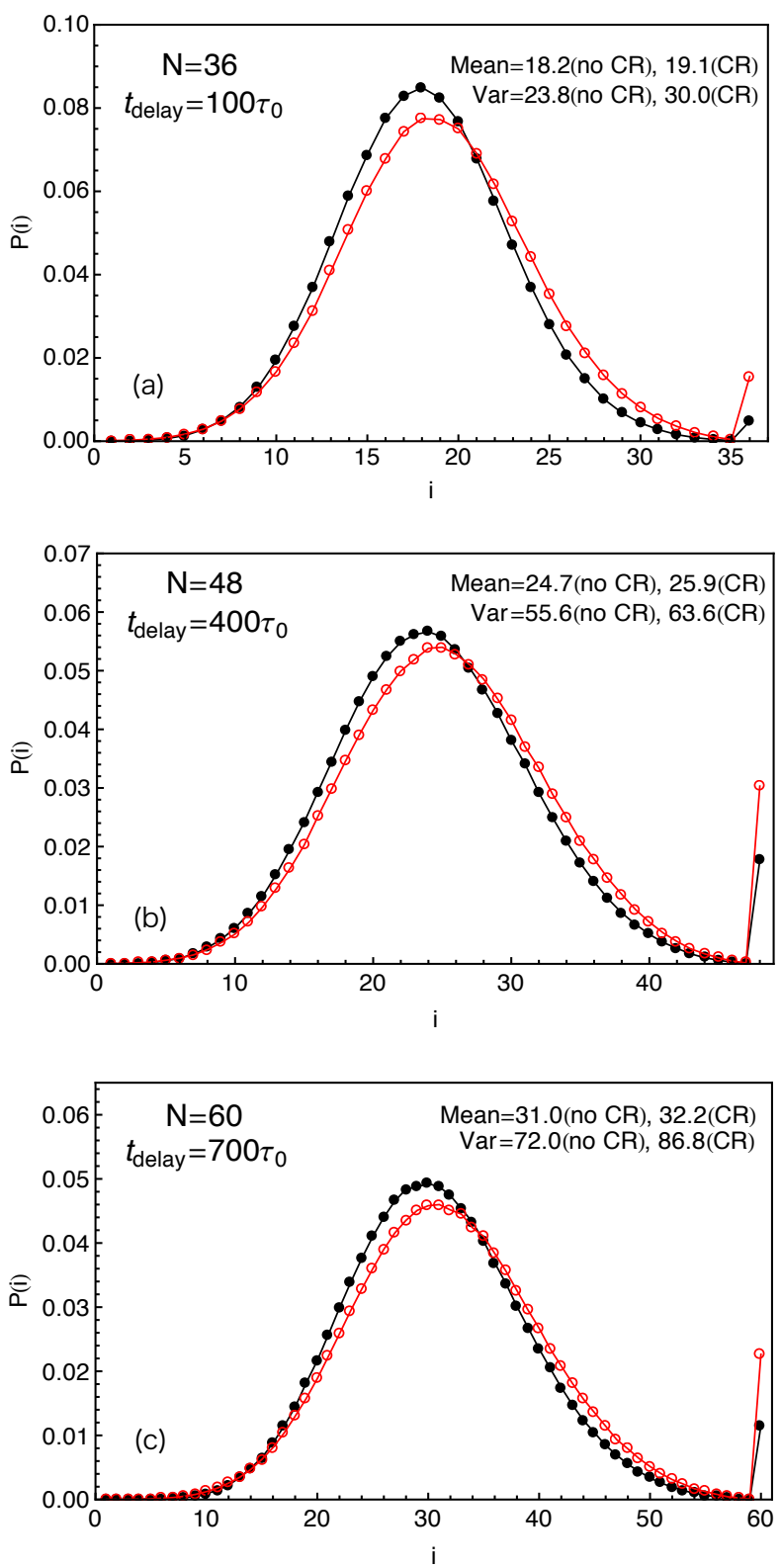

Figure 9: Probability distributions of the locations, as measured by monomer indices, of certain TR slip-links after a delay time $t_{\text {delay }}$ from their initial states of sitting on the middle monomers of the arms $i=N / 2$ at $t=0$. The simulation results are obtained in both the systems with (red circles) and without (black disks) constraint release. The means and variances of these distributions are also provided for reference. 
indices, are then tracked as a function of time. Fig. 9 shows the probability distributions of the locations of the sampled TR slip-links after a carefully chosen delay time $t_{\text {delay }}$ during which nearly all of the sampled slip-links (> 97\%) are able to move along the arms without directly touching the two boundaries. The means and variances of the distribution functions are also provided in the plots. They provide quantitative measurements of the CR effects in two aspects. On the one hand, the continuous creation and destruction of entanglements along the arms lead to large magnitude of fluctuations of the sampled TR slip-links, as reflected in the larger variance of the distributions in the systems with $\mathrm{CR}$ than those in systems without CR. On the other hand, the CR effect is also found to induce a stronger shift of the mean position of the TR slip-links towards the arm ends in comparison with the systems without $\mathrm{CR}$, which would lead to a faster stress relaxation in the star polymer melts. We believe that this CR-induced drifting behavior of entanglements towards arm ends should be taken into account in developing quantitative theories for dynamics of star, or more generally branched, polymers, and so will discuss its physical origin in more details.

\section{Investigating physical origin of the drifting behavior of TR slip-links}

In the single-chain slip-spring model, the entanglements are treated as binary contacts between two different arms such that the CR spectrum is included in a self-consistent manner. This is consistent with the current tube theories which are generally developed on a single molecule basis with self-consistent treatment of contour length fluctuations or arm retraction and constraint release. ${ }^{5,13,16}$ In this section, we propose a simplified single-chain stochastic model which inherits the main features of the single-chain slip-spring model, but is at a more coarse-grained level. It is used to identify the essential physical elements contributing to the drifting behavior of TR entanglements along the primitive paths and so provide useful information for developing quantitative theories of branched polymers. For convenience of 
discussion, we still call entanglements as slip-links in this section.

\subsection{Single-chain stochastic model}

The simplified single-chain stochastic model is constructed using the following protocol.

1) A one-dimensional linear chain is introduced for representing the primitive path of a star arm. This chain is sufficiently long such that during the time window we are interested in, the studied entanglements or slip-links will not be reached by the retracting arm end. The creation and destruction of the slip-links only result from constraint release. This effectively separates the CR effects from the arm retraction dynamics.

2) The length unit of the primitive path is set to be the tube segment length $a$ so that the number density of slip-links along the path is 1 . The slip-links are initially placed at positions $r_{i}=i$ where $i \in \mathbb{N}$. The microstate of a slip-link is defined by its sequential index counting from the origin along the primitive path.

3) The origin of the primitive path is treated as the reflecting boundary representing the branch point. The repulsive interaction between the slip-links and the reflecting boundary is given by a harmonic potential,

$$
U_{w}(r)= \begin{cases}\frac{k_{w}}{2}\left(r-r_{w}\right)^{2}, & \text { for } r<r_{w} \\ 0, & \text { for } r \geq r_{w}\end{cases}
$$

where $r_{w}$ and $k_{w}$ are the cut-off distance and the spring constant of the repulsive wall potential, respectively.

4) The neighboring slip-links are subject to excluded volume interactions to prevent them from crossing each other. The interaction potential takes a harmonic repulsive form,

$$
U_{e x}\left(r_{i}, r_{i+1}\right)= \begin{cases}\frac{k}{2}\left(r_{i+1}-r_{i}-r_{c}\right)^{2}, & \text { for } 0<r_{i+1}-r_{i}<r_{c} \\ 0, & \text { otherwise }\end{cases}
$$


where $r_{c}$ is the cut-off distance of the potential and $k$ is the spring constant. The value of $k$ is chosen to be large enough such that $r_{i+1}$ is always larger than $r_{i}$ during the simulation run, i. e., no swapping of neighboring slip-links. As will be discussed in Sec. 5.3, the introduction of a low swapping rate between neighboring slip-links will only have small quantitative effect on the simulation results.

5) The motion of the slip-links along the primitive path is controlled by the standard stochastic differential equation (SDE)

$$
\xi \frac{d r_{i}}{d t}=-\nabla U\left(r_{i}\right)+W_{i}(t)
$$

where $\xi$ is the friction coefficient of the slip-link and the stochastic force $W$ is a Gaussian white noise

$$
\left\langle W_{i}(t) W_{j}\left(t^{\prime}\right)\right\rangle=2 k_{B} T \xi \delta_{i j} \delta\left(t-t^{\prime}\right)
$$

with $\delta_{i j}$ being the Kronecker delta function and $\delta(t)$ the Dirac delta function, respectively.

6) At the creation of each slip-link, it is assigned a lifetime extracted from a given distribution function. Once its remaining lifetime drops to zero, the slip-link is deleted from the system. Since we simulate the systems in equilibrium, the creation and destruction of slip-links in the system satisfy the detailed balance condition throughout the simulation run.

7) The target TR slip-links are introduced by taking an equilibrium configuration of the system and assigning "very long" lifetimes to a number of selected slip-links such that they will not be deleted from the middle of the primitive path during the time period we are interested in. Due to this biased choice of lifetimes, the TR slip-links themselves do not satisfy the detailed balance condition in the time window we studied, although the whole system is always in equilibrium.

8) In order to keep the number density of slip-links nearly constant, the outer-most $Z$-th slip-link is always subject to a harmonic attractive force from an anchor point at $r=Z$, which effectively restricts its mean position at $r=Z$. In general, we have over a hundred slip-links 
in the system so that the fluctuation of the outer-most slip-link will not affect the dynamics of the slip-links near the branch point over the time of interest.

In the current work, the cut-off distances $r_{w}$ and $r_{c}$ of the repulsive potentials are both set to be 0.5 , and the two spring constants $k$ and $k_{w}$ are set to be 100 . The friction coefficient of the slip-links is $\xi=0.5$ and the time-step size used to integrate the SDEs is $\Delta t=0.005$. The values of $k, k_{w}$ and $\Delta t$ should be determined carefully for preventing the slip-links from crossing each other. Other than that, the use of a different set of parameters will only affect the results quantitatively but not qualitatively.

\subsection{Dynamics in the system with a single TR slip-link}

It is known that in a 1D system consisting of freely diffusing particles the reflecting boundary alone can lead to the shift of the mean positions of the particles away from it. Suppose that there is only a single slip-link along the primitive path. The mean position of this slip-link does not change from its initial location $r$ until it meets the reflecting boundary at a time scale of $t \sim r^{2} / D_{0}$ with the Brownian diffusion coefficient $D_{0}=k_{B} T / \xi$. After that its mean position will drift away from $r$ towards the arm end. More detailed analytical treatment of such a problem can be found in Ref. ${ }^{62}$ The reflecting boundary effect exists in both systems with CR and without CR. In this work we use the simplified single-chain stochastic model to highlight the CR effect that speeds up the drifting of TR slip-links away from the branch point.

In entangled star polymer melts, the relaxation time scales and correspondingly the constraint release events have very broad spectra. We start our study with the simplest case that all slip-links have only one single characteristic relaxation time $\tau_{c}$. Initially 100 slip-links are placed at $x_{i}=i, i \in\{1,2, \cdots, 100\}$ along the primitive path with their lifetimes extracted

from an exponential distribution $\frac{1}{\tau_{c}} \exp \left(-t / \tau_{c}\right)$. At each time step, the probability to insert one slip-link at any location on the segment between $(i-1)$-th and $i$-th slip-links is equal to $\Delta t / \tau_{c}$ such that the detailed balance condition is satisfied. One of the slip-links is chosen as 

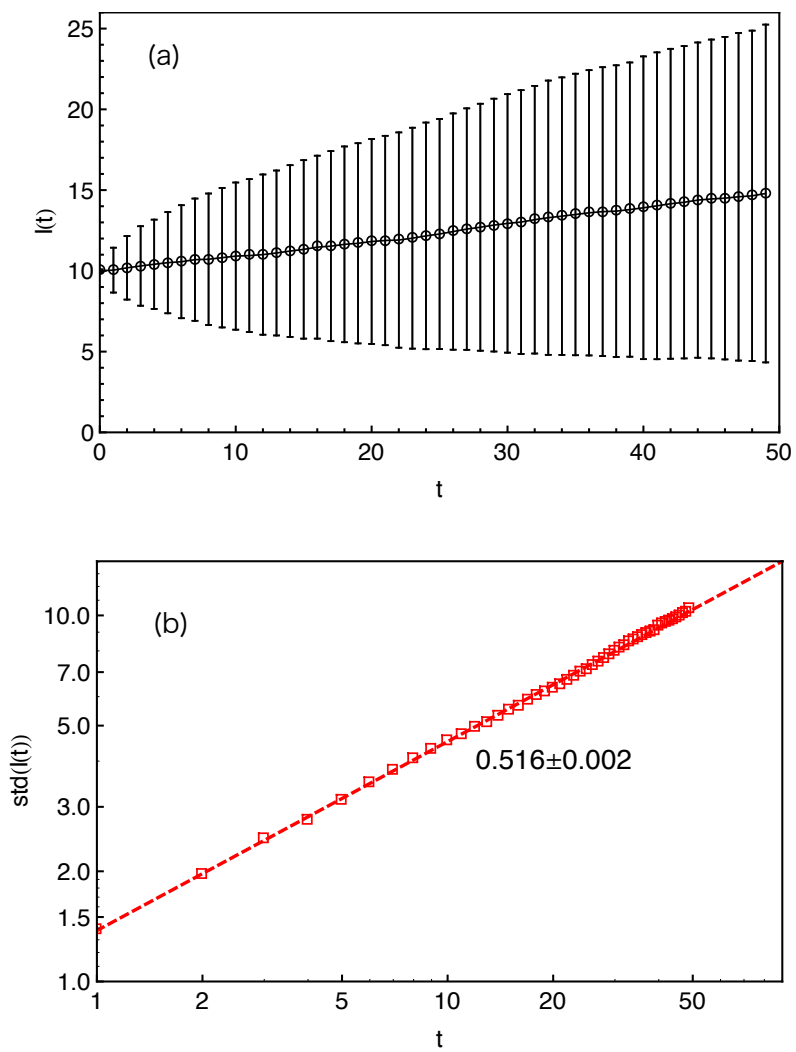

Figure 10: (a) Propagation of the mean sequential index (black circles) and the corresponding standard deviation (vertical bars) of a TR slip-link initially sitting at $r=10$. All other sliplinks have a single characteristic time $\tau_{c}=10$. (b) Log-log plot of the standard deviation in (a). 
the target TR slip-link and assigned a long lifetime (marked as $+\infty$ for convenience) such that it will not be deleted during the time period that we are interested in.

In the following analysis, we track the relative positions of the slip-link along the primitive path by their sequential index counting from 1 for the one closest to the branch point. Fig.10(a) shows the propagation of the mean sequential index of a TR slip-link initially sitting at $i(0)=10$ (and also $r(0)=10$ ) in the system with $\tau_{c}=10$, together with the standard deviation of its position distribution. It can be seen that the mean position of the TR slip-link increases linearly over time, corresponding to a constant drifting velocity. The standard deviation of the position distribution measures the fluctuation amplitude of the TR slip-link over time, which scales as $t^{1 / 2}$, as shown in Fig.10(b) by the log-log plot. Due to the continuous destruction and creation of other slip-links, the position fluctuation of the TR slip-link is apparently of much larger amplitude than that in a system without CR where all slip-links are TR ones and each of them is effectively caged by two nearest neighbors before being deleted from the arm end. In the system studied in Fig. 10, there is no other "long-lived" slip-links in regard to the target TR one, the linear drifting behavior of the target slip-link away from the branch point results from the combined effect of the reflecting boundary condition and the excluded volume interactions between the slip-links. We also found that the slope of the time-dependent mean position line, $i(t)$, or the drifting velocity of the TR slip-link, depends on the characteristic time $\tau_{c}$ of other slip-links. The smaller $\tau_{c}$ leads to higher CR rates and so larger drifting velocity. The high frequency of CR events effectively reduces the friction experienced by the TR slip-link when diffusing along the primitive path and so increases its diffusion coefficient and drifting velocity.

In an equilibrium system the detailed balance condition requires that the numbers of successful moves of the system into and out of any microstate must be equal. In other words, each elementary process should be equilibrated by its reverse process such that no net flux between two microstates. As mentioned above the microstate $S_{i}$ of a slip-link on a given arm is defined by its sequential index $i$ counting from the branch point. If the slip- 
link is deleted from the arm, its microstate is denoted as $S_{\infty}$. Since the whole system is in equilibrium, there is a stationary probability $\pi_{i}$ for finding the slip-link in a microstate $S_{i}$. The detailed balance condition implies that $\pi_{i} W_{i, j}=\pi_{j} W_{j, i}$ where $W_{i, j}$ is the transition probability from microstate $S_{i}$ to $S_{j}$.

After each time step from $t$ to $t+\Delta t$, a slip-link at microstate $S_{i}(t)$ will take one of the four possible moves: moving into one of the two nearest neighboring states $S_{i-1}$ and $S_{i+1}$, remaining in the original state $S_{i}$, or being deleted from the arm $S_{\infty}$ by CR. There are thus only four non-zero transition probabilities which are $W_{i, i-1}, W_{i, i+1}, W_{i, i}$ and $W_{i, \infty}$. It follows from the detailed balance condition that

$$
\begin{aligned}
\pi_{i} W_{i, i+1} & =\pi_{i+1} W_{i+1, i} \\
\pi_{i} W_{i, i-1} & =\pi_{i-1} W_{i-1, i} \\
\pi_{i} W_{i, i} & =\pi_{i} W_{i, i} \\
\pi_{i} W_{i, \infty} & =\pi_{\infty} W_{\infty, i}
\end{aligned}
$$

where $W_{\infty, i}$ and $W_{i, \infty}$ are the probabilities of a slip-link to be added to and deleted from the microstate $S_{i}$, respectively. However, the situation is different for the target TR slip-links. Eqs.4, 5 and 6 are still satisfied, but not Eq.7. Since the TR slip-links can not be deleted from the middle segments of the arm by CR, $W_{i, \infty}$ is zero, while $W_{\infty, i}$ has a positive value. The detailed balance is thus not satisfied for the TR slip-links, although the whole system is in equilibrium. This is the origin of the drifting behavior of these slip-links towards the arm-end, as illustrated in Fig.10(a).

To see how the detailed balance is satisfied in the whole system, we can refer to the more realistic Kremer-Grest bead-spring model and single-chain slip-spring model systems. If a process in which one originally deepest entanglement or slip-link is deleted from the free end of one star arm is observed, there is an equal probability to find a reverse process that a sliplink is added to the free end of the same or any other arm, moves inward towards the branch 
point and is eventually deleted from the arm near the branch point by CR. For calculating the terminal relaxation times of the star polymers, one only considers the previous process where the originally deepest entanglement or tube segment is released by the arm free end. It is for this reason that the drifting behavior of the entanglements or slip-links becomes important and should be taken into account for making quantitative predictions.

\subsection{Dynamics in the system with multiple TR slip-links}

According to the binary picture of entanglements, most of the CR events involve at least one arm free end whose retraction releases the corresponding entanglement. This is especially the case in the single-chain slip-spring model where each slip-link on one arm is coupled with another slip-link on a different arm and the slip-link can be destructed from either one of the two arm ends. Therefore for a given arm, there are on average about half of the entanglements or slip-links destructed from its own free end and so counted as TR slip-links. The dynamics of a target TR slip-link on this arm will surely be affected by the presence of other TR slip-links, particularly the ones in between itself and the branch point. We investigate this effect by using a single-chain system similar to that studied in Sec. 4.2, apart from assigning infinite lifetimes to both the 3rd and 10th (target) slip-links to make them TR ones.

The propagation of the mean sequential index $i(t)$ of the target slip-link is shown in

Fig.11 as squares. In comparison with the system with a single TR slip-link (circles), the $i(t)$ results also demonstrate a linear drifting behavior, but with a slope about twice of that in the previous case. The existence of the "long-lived" slip-links in between the branch point and the target one thus enhances the drifting behavior of the target TR slip-link. We also found that the drifting velocity of the target slip-link is independent of the initial location of the inner TR slip-link (anywhere from 1 to 9). The dynamics of the inner TR slip-link is similar to that of the single TR slip-link studied in Fig.10. Its mean position drifts away from the branch point due to the reflecting boundary effect. Since the target TR slip-link can 
not get any closer to the branch point than the inner TR slip-link due to excluded volume interactions, it effectively feels a continuously shifting reflecting boundary imposed by the drifting inner TR slip-link. The higher drifting velocity of the target TR slip-link can thus be understood as a collective effect of the continuously shifting reflecting boundary towards the arm end and the resulted drifting of the slip-link mean position. This collective effect will become even stronger if there are more TR slip-links in between the branch point and the target one, as shown below.

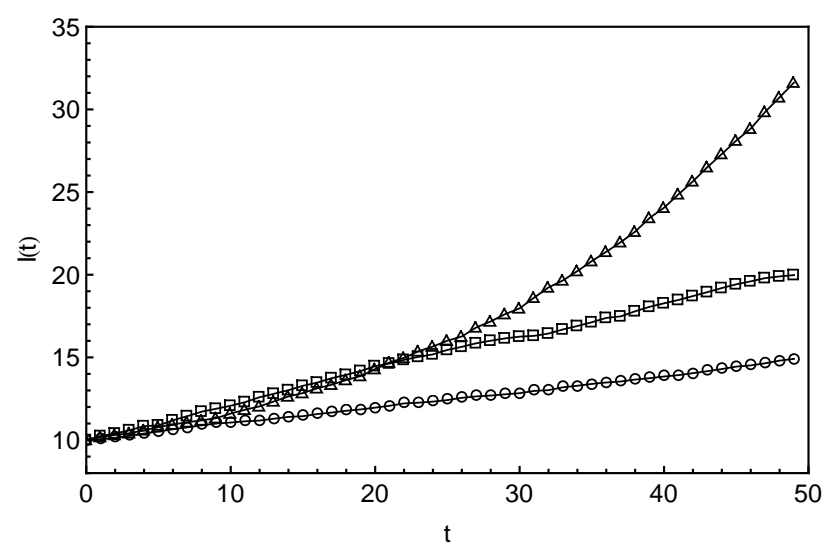

Figure 11: Propagation of the mean sequential index of a target TR slip-link that initially sat at $i(0)=10$ in three different single-chain systems: 1 ) the system with a single TR slip-link (circles, same results as in Fig.10(a)); 2) the system with an extra TR slip-link in between the branch point and the target one (squares); 3) the system with bimodal CR spectrum (triangles)

\subsection{Effect of broad CR spectrum}

The broad CR spectrum in star polymer melts implies that new entanglements or slip-links are continuously created in between the branch points and the target TR entanglements, see Fig. 8. Some of them will live longer than the remaining lifetimes of the target TR ones. For a given TR slip-link, the number of "long-lived" slip-links in between it and the branch point increases with time, as demonstrated in Fig.7. To incorporate this effect into the single-chain stochastic model, we take a bimodal representation of the CR spectrum in which two well-separated characteristic relaxation times of the slip-links are assumed. In 
this work, the lifetimes of $90 \%$ newly created slip-links are extracted from an exponential distribution with the characteristic time $\tau_{c 1}=10$, while the other $10 \%$ extracted from that with $\tau_{c 2}=10^{7}$. The value of $\tau_{c 2}$ is chosen such that the corresponding lifetimes of the sliplinks are long enough for them to be treated as "long-lived" ones for the target TR slip-links during the time period we are interested in.

The initial setup of the single-chain system is again similar to that studied in Sec. 4.2 with the target TR slip-link sitting at $i(0)=10$. The lifetimes of newly created slip-links are now assigned according to the bimodal distributions. Fig.11 shows the propagation of the mean sequential index of the target TR slip-link by triangles. We can see that the slope of the $i(t)$ curve or the drifting velocity of the target TR slip-link is no longer constant, but increases with time. The accelerated motion of the target slip-link towards the arm end clearly results from the continuously increasing number of "long-lived" slip-links created by $\mathrm{CR}$ on the segment between the branch point and the target one. The dynamics of TR entanglements in real entangled star polymer melts should follow the same qualitative behavior, although the CR spectrum there is much broader and more complicated.

Our simulation results thus reveal a relaxation mechanism in which the TR entanglements or slip-links in entangled star polymers experience an accelerated drifting process over time towards the arm free end, which originates from the collective effect of the reflecting boundary (branch point), excluded volume interactions of entanglements and broad CR spectrum in the system. Such relaxation process will surely contribute to the faster relaxation of the star polymers in the systems with CR than those in systems without CR. To the best of our knowledge, this relaxation mechanism has not been addressed in previous published works. 

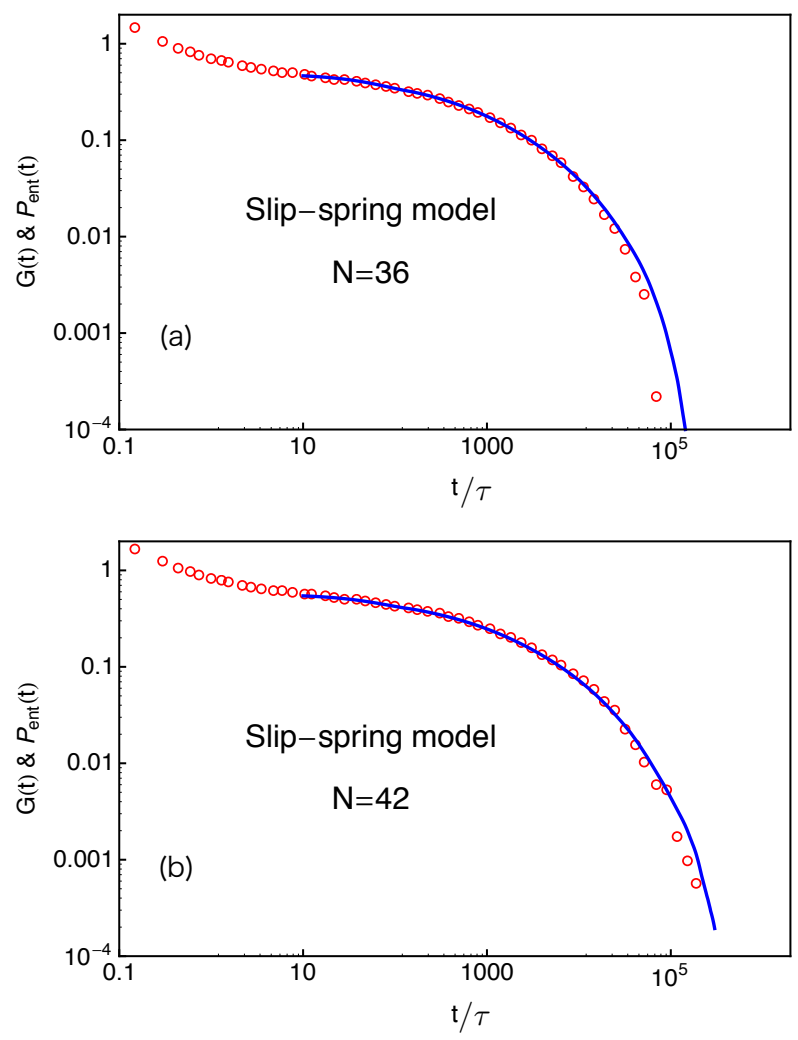

Figure 12: Comparison between the stress relaxation function $G(t)$ (red circles) and survival probability function of slip-links $P_{\text {ent }}(t)$ (blue curves) obtained in the slip-spring model simulations of star polymers with two different arm-lengths: (a) $N=36$ and (b) $N=42$. The $P_{\text {ent }}(t)$ curves have been shifted vertically. 


\section{Further discussions}

\subsection{Reproducing dynamic observables in single-chain slip-spring model}

We have shown in Sec. 2.4 that the stress relaxation function $G(t)$ in the star polymers represented by the Kremer-Grest bead-spring model can be reproduced reasonably well by calculating the survival probability $P_{\text {ent }}(t)$ of all persistent close-contacts between the mean paths of the star arms, see Fig.4. The same type of analysis can be carried out for the single-chain slip-spring model simulations. Since we have saved the trajectory of each sliplink during its lifetime, it is straightforward to calculate $P_{\text {ent }}(t)$ of the slip-links using Eq.3 and compare the results with the stress relaxation function of the system. As mentioned in Sec. 3, the $G(t)$ data in the slip-spring model are obtained from the single-chain stress autocorrelation functions. It has been shown that the cross-correlation contribution can count to $30-50 \%$ of the total stress modulus, depending on the simulation model used. ${ }^{30,59,60}$ But since the stress auto-correlation function is proportional to the total stress relaxation function at large time scales, the comparison between $G(t)$ and $P_{\text {ent }}(t)$ is not affected by the use of the singe-chain auto-correlation function. Fig.12 shows that a very good agreement has been achieved between the $G(t)$ (red circles) and vertically shifted $P_{\text {ent }}(t)$ (blue curves) data for two different arm lengths. This further verifies the observation in Fig.4 that the terminal stress relaxation of entangled polymers can be well described by the survival probability of all entanglements or slip-links in the system.

According to the Doi-Edwards theory for polymers in a fixed network, ${ }^{2}$ the tube survival

probability function $\mu(t)$ is proportional to the correlation function of the chain end-to-end vector $\boldsymbol{R}_{e}$ as

$$
\varphi(t)=\left\langle\boldsymbol{R}_{e}(t) \cdot \boldsymbol{R}_{e}(0)\right\rangle /\left\langle\boldsymbol{R}_{e}^{2}(0)\right\rangle
$$

On the other hand, since entangled star polymer melts exhibit broad spectrum of relaxation 


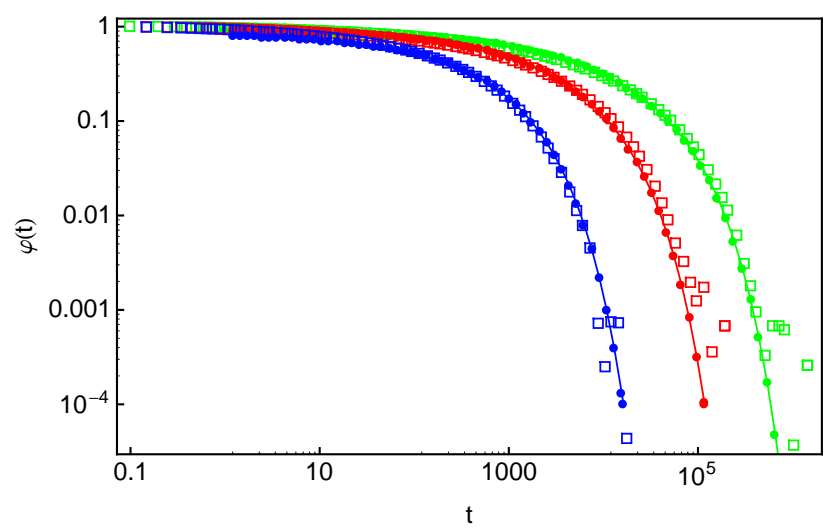

Figure 13: Comparison between the tube survival probability function $\mu(t)$ calculated using Eq.8 (disks) and arm end-to-end vector correlation function $\varphi(t)$ (open squares) obtained in the slip-spring simulations of star polymers with arm lengths $N=24$ (blue), 36(red) and 48(green), respectively.

time scales, $\mu(t)$ can be conveniently estimated using the mean first passage time $\tau_{c r}(s)$ as follows,

$$
\mu(t)=\int_{0}^{1} d s \int_{t}^{+\infty} f\left(s, t^{\prime}\right) d t^{\prime}=\int_{0}^{1} \exp \left(-\frac{t}{\tau_{c r}(s)}\right) d s
$$

supposing that the FP time distribution $f\left(s, t^{\prime}\right)$ of tube segment $s$ has an exponential form $\exp \left(-\frac{t^{\prime}}{\tau_{c r}(s)}\right) / \tau_{c r}(s)$ for every $s$ value. ${ }^{3,15}$ In the single-chain slip-spring model simulations we found that $f\left(s, t^{\prime}\right)$ actually deviates from the exponential distribution due to constraint release. More detailed discussion about this deviation will be left for a future publication. ${ }^{63}$ In the current work we use Eq.8 as an empirical approach for calculating the tube survival probability function from the mean FP times of slip-links. The $\mu(t)$ results calculated using only contributions from the TR slip-links are presented in Fig.13 for three different arm lengths. The good agreement between $\mu(t)$ and $\varphi(t)$ in all three cases at least provides semi-quantitative support for the idea that the dielectric relaxation function, or arm end-toend vector correlation function, in star polymers is determined by the relaxation of the TR entanglements or slip-links which are deleted from the free end of the arm they sat on. 


\subsection{Possible theoretical model for describing dynamics in entangled star polymers}

Considering the limitation of the DTD model, one may seek to develop a more detailed single-molecule model that can simultaneously predict the interested dynamic properties, such as the first passage times of TR entanglements and the dielectric and stress relaxation functions, of star and other branched polymers. Based on the findings of this work, it is possible to construct a single-arm model which contains a set of stochastic differential equations for describing the dynamics of the arm free end and TR entanglements. The first SDE describes the arm retraction process where the arm free end fluctuates under an effective potential similar to that given by the Pearson-Helfand model. ${ }^{10}$ The other SDEs are equations of motion of the TR entanglements which experience a prescribed velocity field and so drift towards the arm end as represented by biased fluctuations. Once one TR entanglement meets the arm-end, its FP time is recorded and will later be used for predicting other dynamic properties. The treatment of the velocity field should incorporate the collective contributions from the reflecting boundary at branch point, excluded volume interactions between entanglements and constraint release effect. The exact expression of this field is still unknown. At least in the single-chain slip-spring model, the velocity of the TR slip-link drift is found to increase with time in an exponential form when approaching the arm-end, ${ }^{63}$ which can also be seen in the simulation results of the simplified single-chain model with bimodal CR spectrum (triangles) in Fig. 11.

\subsection{Examining dynamics of entanglements in other simulation mod- els}

In our single-chain slip-spring and simplified single-chain stochastic models, the entanglements or slip-links are not allowed to pass over each other or occupy the same monomer, which effectively introduces excluded volume interactions between entanglements. A similar 
approach has been used by Shanbhag et al. in their dual slip-link model where the slip-links do not change their relative ordering along the primitive paths. ${ }^{25}$ This assumption is partly supported by the low swapping rate between neighboring entanglements as found in the persistent close-contact analysis of star polymers represented by the Kremer-Grest bead-spring model (see Sec.2.4.).

In order to examine the effect of entanglement swapping or reordering along the primitive path, we modify the simplified single-chain stochastic model by allowing the slip-links to cross each other with a probability comparable to that found in MD simulations using the Kremer-Grest model. According to the parameters used in the simplified single-chain model, the unit time scale is the time required for a slip-link to explore a distance of one tube segment (one unit length) along the primitive path, which is about the Rouse relaxation time of an entanglement strand $\tau_{e}$. Thus the probability for each slip-link (including the TR ones) to swap with its nearest neighbor in a unit time is set to be $1 \%$ in consistence with the swapping rate of once per $100 \tau_{e}$ found in the Kremer-Grest model. Fig.14 shows the propagation of the mean sequential index of the target TR slip-link (filled triangles), together with the results obtained without swapping (open triangles), for the systems with bimodal CR spectrum. It can be seen that the dynamics of the target TR slip-link is insensitive to the swapping of the slip-links, at least not at the rate we studied. Very similar results have been observed in the simulations of other simplified single-chain stochastic model systems. In principle, we can also introduce the slip-link swapping mechanism into the single-chain slip-spring model. But this will involve very delicate calibration of system parameters, which is beyond the scope of the current work and will be left for later study.

In recent years a number of single- and multi-chain slip-link or slip-spring models have been developed for studying the dynamics of entangled polymers. ${ }^{60,64-71}$ Among them several multi-chain slip-spring models have allowed the slip-links to cross over each other. ${ }^{60,67-69}$ In order to maintain the homogeneous distributions of slip-links in space, additional shortrange repulsions among all beads of the Rouse chains have been introduced. ${ }^{60,67}$ It would 


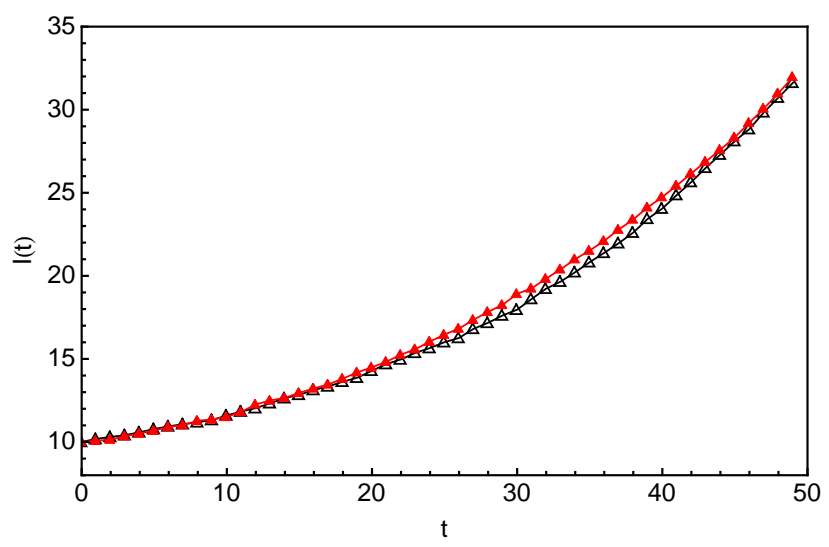

Figure 14: Propagation of the mean sequential index of a target TR slip-link initially sitting at $i(0)=10$ in the simplified single-chain stochastic model systems with bimodal CR spectrum where the slip-links are either allowed to swap with their nearest neighbors at a rate of $1 \%$ per unit time (filled triangles) or not allowed to cross each other (open triangles, same results as in Fig.10(a)).

be interesting to examine the motion or change of relative ordering of slip-links along the primitive paths in such models, especially for the star polymers. Since it is entropically unfavorable to have several slip-links to sit on the same bead or be closely packed to form a cluster in space, the insertion of a long-lived new entanglement in the middle of an arm or chain will cause the local re-arrangement of the neighboring slip-links in order to re-establish a homogenous distribution of entanglements along the primitive path. This redistribution process of slip-links is entropically driven, regardless whether they can pass over each other or not. As a consequence, the existing slip-links will be effectively pushed towards the free chain ends. The CR-induced drifting behavior of entanglements is thus also expected in these multi-chain slip-spring models. The key issue is that the friction coefficient of the slip-links should be relatively large so that the diffusion of the slip-links along the chain is not overtaking the equilibration process of their local distribution. The magnitude of the drifting effect would depend on the detailed set-up of individual models. It has been noticed that the dynamical constraints imposed by the slip-springs in some of the multichain slip-spring models ${ }^{60,67}$ are softer than that in the single-chain slip-spring model we used. Another interesting topic would be what is the exact physical meaning of the slip- 
slinks in relation to entanglements in real polymer systems, namely are they supposed to represent the topological constraints on polymer dynamics in the general or tube-like sense or to directly mimic the binary entanglements or persistent close contacts between polymer chains as found in the more detailed Kremer-Grest model?

\section{Conclusions}

We presented a multi-scale simulation study of the microscopic picture of constraint release effects in entangled symmetric star polymer melts. In molecular dynamics simulations using the Kremer-Grest bead-spring model, entanglement dynamics was investigated by analyzing the persistent close contacts between pairs of primitive paths of neighboring polymer strands. The original contact map algorithm developed by Likhtman and Ponmurugan ${ }^{27}$ was updated by taking into account the contributions from temporarily disappeared closecontacts involving middle segments of both strands. For both linear and symmetric star polymers, the resulting survival probabilities of these determined close-contacts were found to be in reasonably good agreement with the stress relaxation functions of these systems, indicating that entanglements can be reasonably described by the binary contact picture. It thus allows us to investigate the entanglement dynamics at larger time and length scales by using the single-chain slip-spring model, in which entanglements are treated as paired slip-links between two different chains or arms. In both MD and slip-spring model simulations, we verified the mechanism proposed by Shanbhag et al. ${ }^{25}$ that new entanglements inserted between the branch points and originally deepest entanglements push the deepest entanglements outwards such that they are destructed by relatively shallow arm-retractions.

Furthermore, we found that only entanglements destroyed from arm-ends, termed as Tube Representative (TR) entanglements, contribute to the dielectric or arm end-to-end vector relaxation functions, while for stress relaxation all entanglements contribute. Once the TR entanglements are selected as investigated objects, the combined effects from the reflecting 
boundary at the branch points, the excluded volume interactions between entanglements and the broad CR spectrum produce an acceleration field to drive the mean positions of the entanglements move towards the arm free ends, which speed up the relaxation of the system in comparison with that in systems without CR. A simplified single-chain stochastic model has been developed to clearly demonstrate this combined effect. This microscopic mechanism of CR effects in the relaxation of star polymers has not been addressed before, and should be taken into account for developing quantitative theories for describing the dynamics of entangled branched polymers.

\section{Acknowledgement}

We thank Alexei E. Likhtman for very extensive and fruitful discussions about this work. We also thank Scott T. Milner, Daniel J. Read and Tom C. B. McLeish for stimulating discussions. This work was supported by the Engineering and Physical Sciences Research Council (EPSRC), Grant EP/K017683. We also acknowledge the financial support from the collaboration funds of the State Key Laboratory of Polymer Physics and Chemistry, Chinese Academy of Sciences, Beijing, China.

\section{References}

(1) de Gennes, P. G. Reptation of a polymer chain in the presence of fixed obstacles. J. Chem. Phys. 1971, 55, 572.

(2) Doi, M.; Edwards, S. The Theory of Polymer Dynamics; Oxford University Press, 1988.

(3) McLeish, T. C. B. Tube theory of entangled polymer dynamics. Adv. Phys. 2002, 51, $1379-1527$.

(4) Rubinstein, M.; Colby, R. H. Self-consistent theory of polydisperse entangled polymers — linear viscoelasticity of binary blends. J. Chem. Phys. 1988, 89, 5291-5306. 
(5) Likhtman, A. E.; McLeish, T. C. B. Quantitative theory for linear dynamics of linear entangled polymers. Macromolecules 2002, 35, 6332-6343.

(6) Milner, S. T.; McLeish, T. C. B.; Likhtman, A. E. Microscopic theory of convective constraint release. J. Rheol. 2001, 45, 539-563.

(7) Graham, R. S.; Likhtman, A. E.; McLeish, T. C. B.; Milner, S. T. Microscopic theory of linear, entangled polymer chains under rapid deformation including chain stretch and convective constraint release. J. Rheol. 2003, 47, 1171.

(8) Likhtman, A. E.; Graham, R. S. Simple constitutive equation for linear polymer melts derived from molecular theory: Rolie-Poly equation. J. Non-Newtonian Fluid Mech. 2003, 114, 1-12.

(9) Qin, J.; Milner, S. T. Tube Dynamics Works for Randomly Entangled Rings. Phys. Rev. Lett. 2016, 116, 068307.

(10) Pearson, D. S.; Helfand, E. Viscoelastic Properties of Star-sahped Polymers. Macromolecules 1984, 17, 888-895.

(11) Ball, R.; McLeish, T. Dynamics dilution and the viscosity of star polymer melts. Macromolecules 1989, 22, 1911-1913.

(12) Marrucci, G. RELAXATION BY REPTATION AND TUBE ENLARGEMENT - A MODEL FOR POLYDISPERSE PolymerS. J. Polym. Sci., Polym. Phys. Ed. 1985, 23, 159-177.

(13) Milner, S. T.; McLeish, T. C. B. Parameter-free theory for stress relaxation in star polymer melts. Macromolecules 1997, 30, 2159-2166.

(14) Milner, S. T.; McLeish, T. C. B. Reptation and contour-length fluctuations in melts of linear polymers. Phys. Rev. Lett. 1998, 81, 725-728. 
(15) Hanggi, P.; Talkner, P.; Borkovec, M. Reaction-rate Theory - 50 Years after Kramers. Rev. Mod. Phys. 1990, 62, 251-341.

(16) Larson, R. G. Combinatorial Rheology of Branched Polymer Melts. Macromolecules 2001, 34, 4556-4571.

(17) Park, S.; Shanbhag, S.; Larson, R. A hierarchical algorithm for predicting the linear viscoelastic properties of polymer melts with long-chain branching. Rheol. Acta 2005, 44, 319-330.

(18) Wang, Z.; Chen, X.; Larson, R. G. Comparing tube models for predicting the linear rheology of branched polymer melts. J. Rheol. 2010, 54, 223-260.

(19) Das, C.; Inkson, N. J.; Read, D. J.; Kelmanson, M. A.; McLeish, T. C. B. Computational linear rheology of general branch-on-branch polymers. J. Rheol. 2006, 50, 207-234.

(20) van Ruymbeke, E.; Bailly, C.; Keunings, R.; Vlassopoulos, D. A general methodology to predict the linear Rheology of branched Polymers. Macromolecules 2006, 39, 62486259.

(21) Cao, J.; Zhu, J.; Wang, Z.; Likhtman, A. E. Large deviations of Rouse polymer chain: First passage problem. J. Chem. Phys. 2015, 143, 204105.

(22) Watanabe, H.; Matsumiya, Y.; Osaki, K. Tube dilation process in star-branched cispolyisoprenes. J. Polym. Sci., Part B: Polym. Phys. 2000, 38, 1024-1036.

(23) Matsumiya, Y.; Watanabe, H. Further Test of the Tube Dilation Process in StarBranched cis-Polyisoprene:âĂL' Role of Branching-Point Fluctuation. Macromolecules 2001, 34, 5702-5710.

(24) Watanabe, H.; Matsumiya, Y.; Inoue, T. Dielectric and viscoelastic relaxation of highly entangled star polyisoprene: Quantitative test of tube dilation model. Macromolecules 2002, 35, 2339-2357. 
(25) Shanbhag, S.; Larson, R.; Takimoto, J.; Doi, M. Deviations from dynamic dilution in the terminal relaxation of star polymers. Phys. Rev. Lett. 2001, 87, 195502.

(26) Kremer, K.; Grest, G. Dynamics of Entangled Linear Polymer Melts - A Molecular Dynamics Simulation. J. Chem. Phys. 1990, 92, 5057-5086.

(27) Likhtman, A. E.; Ponmurugan, M. Microscopic Definition of Polymer Entanglements. Macromolecules 2014, 4\%, 1470-1481.

(28) Likhtman, A. E. Single-chain slip-link model of entangled polymers: Simultaneous description of neutron spin-echo, rheology, and diffusion. Macromolecules 2005, 38, 61286139.

(29) Likhtman, A. E.; Sukumaran, S. K.; Ramirez, J. Linear Viscoelasticity from Molecular Dynamics Simulation of Entangled Polymers. Macromolecules 2007, 40, 6748-6757.

(30) Cao, J.; Likhtman, A. E. Time-Dependent Orientation Coupling in Equilibrium Polymer Melts. Phys. Rev. Lett. 2010, 104, 207801.

(31) Wang, Z.; Likhtman, A. E.; Larson, R. G. Segmental Dynamics in Entangled Linear Polymer Melts. Macromolecules 2012, 45, 3557-3570.

(32) Zhou, Q.; Larson, R. G. Direct Molecular Dynamics Simulation of Branch Point Motion in Asymmetric Star Polymer Melts. Macromolecules 2007, 40, 3443-3449.

(33) Bacova, P.; Hawke, L. G. D.; Read, D. J.; Moreno, A. J. Dynamics of Branched Polymers: A Combined Study by Molecular Dynamics Simulations and Tube Theory. Macromolecules 2013, 46, 4633-4650.

(34) Bacova, P.; Moreno, A. J. Real-Space Analysis of Branch Point Motion in Architecturally Complex Polymers. Macromolecules 2014, 47, 6955-6963. 
(35) Bacova, P.; Lentzakis, H.; Read, D. J.; Moreno, A. J.; Vlassopoulos, D.; Das, C. BranchPoint Motion in Architecturally Complex Polymers: Estimation of Hopping Parameters from Computer Simulations and Experiments. Macromolecules 2014, 47, 3362-3377.

(36) Bisbee, W.; Qin, J.; Milner, S. T. Finding the tube with isoconfigurational averaging. Macromolecules 2011, 44, 8972-8980.

(37) Cao, J.; Qin, J.; Milner, S. T. Simulating Constraint Release by Watching a Ring Cross Itself. Macromolecules 2014, 47, 2479-2486.

(38) Qin, J.; Milner, S. T. Tubes, Topology, and Polymer Entanglement. Macromolecules 2014, 47, 6077-6085.

(39) Cao, J.; Qin, J.; Milner, S. T. Finding Entanglement Points in Simulated Polymer Melts. Macromolecules 2015, 48, 99-110.

(40) Kröger, M.; Loose, W.; Hess, S. Rheology and Structural-Changes of Polymer Melts via Nonequilibrium Molecular Dynamics. J. Rheol. 1993, 37, 1057-1079.

(41) Kröger, M.; Hess, S. Rheological evidence for a dynamical crossover in polymer melts via nonequilibrium molecular dynamics. Phys. Rev. Lett. 2000, 85, 1128-1131.

(42) Cao, J.; Likhtman, A. E. Shear Banding in Molecular Dynamics of Polymer Melts. Phys. Rev. Lett. 2012, 108, 028302.

(43) Cao, J.; Likhtman, A. E. Simulating startup shear of entangled polymer melts. ACS Macro Lett. 2015, 4, 1376-1381.

(44) Masubuchi, Y.; Watanabe, H. Stress-Optical Relationship in Bead-Spring Simulations for Entangled Polymers under Start-up Shear Flows. Nihon Reoroji Gakkaishi 2016, $44,65-68$. 
(45) Everaers, R.; Sukumaran, S. K.; Grest, G. S.; Svaneborg, C.; Sivasubramanian, A.; Kremer, K. Rheology and microscopic topology of entangled polymeric liquids. Science 2004, 303, 823-826.

(46) Ramírez, J.; Sukumaran, S. K.; Vorselaars, B.; Likhtman, A. E. Efficient on the fly calculation of time correlation functions in computer simulations. J. Chem. Phys. 2010, 133.

(47) Sukumaran, S. K.; Grest, G. S.; Kremer, K.; Everaers, R. Identifying the primitive path mesh in entangled polymer liquids. J. Polym. Sci., Part B: Polym. Phys. 2005, 43, 917-933.

(48) Kröger, M. Shortest multiple disconnected path for the analysis of entanglements in two- and three-dimensional polymeric systems. Comput. Phys. Commun. 2005, 168, 209-232.

(49) Tzoumanekas, C.; Theodorou, D. N. Topological analysis of linear polymer melts: a statistical approach. Macromolecules 2006, 39, 4592-4604.

(50) Anogiannakis, S. D.; Tzoumanekas, C.; Theodorou, D. N. Microscopic Description of Entanglements in Polyethylene Networks and Melts: Strong, Weak, Pairwise, and Collective Attributes. Macromolecules 2012, 45, 9475-9492.

(51) Zhou, Q.; Larson, R. G. Primitive path identification and statistics in molecular dynamics simulations of entangled polymer melts. Macromolecules 2005, 38, 5761-5765.

(52) Read, D. J.; Jagannathan, K.; Likhtman, A. E. Entangled polymers: constraint release, mean paths, and tube bending energy. Macromolecules 2008, 41, 6843-6853.

(53) Doi, M.; Edwards, S. F. Dynamics of concentrated polymer systems. Part 1.-Brownian motion in the equilibrium state. J. Chem. Soc., Faraday Trans. 2 1978, 74, 1789-1801. 
(54) Sukumaran, S. K.; Likhtman, A. E. Modeling Entangled Dynamics: Comparison between Stochastic Single-Chain and Multichain Models. Macromolecules 2009, 42, 43004309.

(55) Shivokhin, M. E.; van Ruymbeke, E.; Bailly, C.; Kouloumasis, D.; Hadjichristidis, N.; Likhtman, A. E. Understanding Constraint Release in Star/Linear Polymer Blends. Macromolecules 2014, 47, 2451-2463.

(56) Ramírez, J.; Sukumaran, S. K.; Likhtman, A. E. Hierarchical Modeling of Entangled Polymers. Macromol. Symp. 2007, 252, 119-129.

(57) Likhtman, A. E. Viscoelasticity and Molecular Rheology. In Comprehensive Polymer Science, 2nd ed.; Elsevier: Amsterdam, 2011.

(58) Masubuchi, Y.; Yaoita, T.; Matsumiya, Y.; Watanabe, H. Primitive chain network simulations for asymmetric star polymers. J. Chem. Phys. 2011, 134.

(59) Ramírez, J.; Sukumaran, S. K.; Likhtman, A. E. Significance of cross correlations in the stress relaxation of polymer melts. J. Chem. Phys. 2007, 126, 244904.

(60) Uneyama, T.; Masubuchi, Y. Multi-chain slip-spring model for entangled polymer dynamics. J. Chem. Phys. 2012, 137, 154902.

(61) Cao, J.; Wang, Z.; Likhtman, A. E. Determining Tube Survival Function through FirstPassage Time of Star Polymers in Network. to be submitted

(62) Redner, S. A Guide to First-Passage Processes; Cambridge University Press, 2001.

(63) Cao, J.; Wang, Z. Manuscript in preparation

(64) Masubuchi, Y.; Takimoto, J.-I.; Koyama, K.; Ianniruberto, G.; Marrucci, G.; Greco, F. Brownian simulations of a network of reptating primitive chains. J. Chem. Phys. 2001, 115, 4387-4394. 
(65) Masubuchi, Y.; Ianniruberto, G.; Greco, F.; Marrucci, G. Entanglement molecular weight and frequency response of sliplink networks. J. Chem. Phys. 2003, 119, 69256930.

(66) Nair, D. M.; ; Schieber, J. D. Linear Viscoelastic Predictions of a Consistently Unconstrained Brownian Slip-Link Model. Macromolecules 2006, 39, 3386-3397.

(67) Chappa, V. C.; Morse, D. C.; Zippelius, A.; Müller, M. Translationally Invariant SlipSpring Model for Entangled Polymer Dynamics. Phys. Rev. Lett. 2012, 109, 148302.

(68) Langeloth, M.; Masubuchi, Y.; Böhm, M. C.; Müller-Plathe, F. Recovering the reptation dynamics of polymer melts in dissipative particle dynamics simulations via slipsprings. J. Chem. Phys. 2013, 138, 104907.

(69) Ramírez-Hernandez, A.; Detcheverry, F. A.; Peters, B. L.; Chappa, V. C.; Schweizer, K. S.; Müller, M.; de Pablo, J. J. Dynamical Simulations of Coarse Grain Polymeric Systems: Rouse and Entangled Dynamics. Macromolecules 2013, 46, 62876299.

(70) Masubuchi, Y.; Watanabe, H. Origin of Stress Overshoot under Start-up Shear in Primitive Chain Network Simulation. ACS Macro Lett. 2014, 3, 1183-1186.

(71) Ramírez-Hernández, A.; Peters, B. L.; Andreev, M.; Schieber, J. D.; de Pablo, J. J. A multichain polymer slip-spring model with fluctuating number of entanglements for linear and nonlinear rheology. J. Chem. Phys. 2015, 143, 243147. 


\section{Graphical TOC Entry}

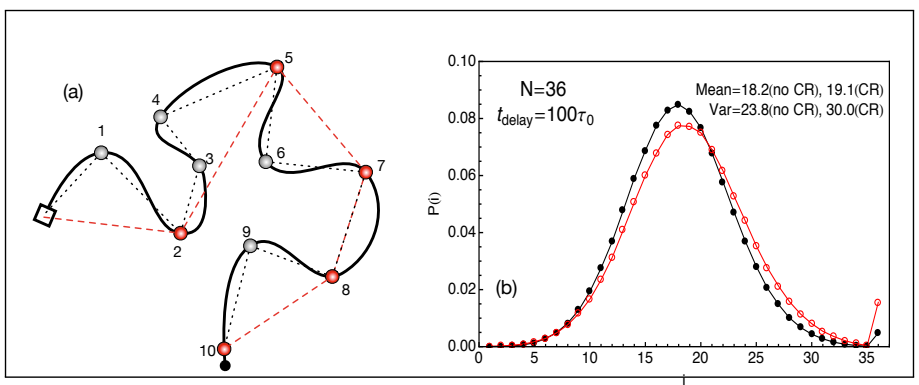

